\title{
Magnetotransport in nearly antiferromagnetic metals
}

\author{
A. Rosch \\ Center for Materials Theory, Department of Physics and Astronomy, Rutgers University, Piscataway, NJ 08854, USA
}

(July 28, 2021)

\begin{abstract}
We present a theory of the magnetotransport in weakly disordered metals close to an antiferromagnetic quantum-critical point. The anisotropic scattering from critical spin fluctuations is strongly influenced by weak but isotropic scattering from small amounts of disorder. This leads to a large regime where the resistivity obeys a scaling form $\rho=\rho_{0}+\Delta \rho \approx \rho_{0}+T^{3 / 2} f\left(T / \rho_{0},\left(p-p_{c}\right) / \rho_{0}, B / \rho_{0}^{3 / 2}\right)$, where $\rho_{0}$ is the residual resistivity, $B$ the magnetic field and $p-p_{c}>0$ measures the distance from the quantum-critical point on the paramagnetic side of the phase diagram. Orbital effects of the magnetic field are most pronounced in very clean samples for not too low temperatures, where the resistivity for increasing magnetic field crosses over from a linear temperature dependence $\Delta \rho \sim T \sqrt{\rho_{0}}$ to a resistivity linear in $B$ and independent of $T$ and $\rho_{0}$. At higher magnetic fields $\Delta \rho$ saturates at a value proportional to $T^{1.5}$ or $T^{2} /\left(p-p_{c}\right)$. Deviations from scaling, the interplay of orbital and spin contributions of the magnetic field and experimental test of the spin-fluctuation model are discussed in detail.
\end{abstract}

72.10.Di,75.30.Mb, 71.27.+a,75.50.Ee

\section{INTRODUCTION}

One of the most important unresolved questions in the field of strongly correlated metals is the stability of the Fermi liquid. In one dimension, the Fermi liquid is destroyed by strong quantum fluctuations. The strange properties of the normal phase of high-temperature superconductors $₫$ as well as the observation of an tnexpected metal-insulator transition in Si-MOSFET 2 a 3 and other systems have cast doubts on the applicability of Landau's Fermi-liquid paradigm in two dimensions.

Even in three dimensions $(3 d)$ a number of heavyfermion metals near an antiferromagnetic (AFM) quantum critical point (QCP) were shown to display striking deviations from conventional Fermi-liquid behavion 313 . The main aim of this paper is to develop a detailed transport theory which allows to decide experimentally whether the non-Fermi liquid behavior near the QCP in weakly disordered metals can be explained by a nearly AFM Fermi liquid.

It is believed that the magnetic transition in heavy Fermion systems results from the competition between the Kondo screening of magnetic moments and the AFM correlations induced by the RKKY interaction 14. Pressure, magnetic fields or doping can influence this interplay of screening and magnetism. This allows to fine-tune systems directly to the QCP where the strongest nonFermi liquid (NFL) effects are found; a schematic phase diagram is shown in Fig. 1. In particular, the resistivity rises as a function of temperature with exponents smaller than 2, the specific heat coefficient diverges or shows a $\sqrt{T}$ cusp, and the susceptibility shows anomalous corrections of the form $T^{\alpha}$, with $\alpha<1$. We stress that both magnetic and non-magnetic phases are heavy-Fermion metals which display, for example, the characteristically large specific heat. The discussion of this paper is restricted to the behavior in the paramagnetic phase close to the QCP.

Despite the growing amount of experimental data, a common agreement on the origin of the observed nonFermi liquid behavior is still lacking. At present it is unclear, whether a single mechanism is responsible for the observed behavior as different exponents have been reported in various compounds. At least three different theoretical scenarios have been proposed and are discussed in literature.

The first scenario is based on the assumption that in a heavy Fermion system below a scale $T_{K}$ the low energy excitations are (heavy) quasi particles and their collective excitations. In this case the QCP should be in the same universality class as the weak-coupling spin-density waw (SDW) transition in a Fermi liquid studied by Hertz 15 17. More precisely, the so-called non-Fermi liquid behavior near the QCP is determined by the mutual interaction between Landau damped spin fluctuations and inelastically scattered quasi-particles in a nearly antiferromagnetic Fermi liquid.

Alternatively, one can envision a situation where the Kondo effects breaks down directly at the transition, e.g. due to strong magnetic fluctuations 18 21. A Fermi liquid description of the transition is then not possible and it has been speculated that fogluations play the dominant role in this scenarid 1920.

Independent of the precise nature of the QCP, sufficiently strong disorder will certainly change the nature of the transition. Indeed, if typical fluctuations of the effective Neèl temperature due to disorder are large compared to the distance of the quantum critical point (Harris criterion), disorder effects are important. Perhaps more importantly, even at some distance form the QCP, in the non-magnetic phase, rare configurations of impurities can lead to a small magnetically ordered regions which can dominate some of the thermodynamic properties in a finite region around the QCP (Griffiths-McCoy 
singularities) 2224.

Up to now, a comprehensive theoretical description exists only for the weak-coupling spin-density wave transition 15. 16. As this theory is above its upper critical dimension, essentially all low-energy properties can be determined analytically. It was realized only recently 2527 that the calculation of transpert quantities is quite subtle and earlier predictions 12 27 are not valid in weakly disordered materials. The complications arise because the scattering from spin-waves is extremely anisotropic and effective only in small areas on the Fermi surface. Therefore the transport properties strongly depend both qualitatively and quantitatively on how other scattering mechanisms redistribute quasi particles and scatter them into these small regions.

The resistivity is often the most sensitive experimental probe to study the QCP, and in a number of (pressure tuned) systems it is the only available quantity. As the effects of strong disorder are poorly understood at present, it is important to study very clean systems. The goal of this paper is therefore to establish a set of predictions for the resistivity within a SDW approach for weakly disordered metals close to an AFM QCP. The interplay of the relevant scattering mechanisms is studied within a semi-classical approach using a Boltzmann equation. The model and the Boltzmann equation is defined in section II A. In section IIB the analytic solution of the Boltzmann equation is presented in the limit of low temperature and weak, but finite disorder. The scaling properties close to the quantum critical point are emphasized and numerical solutions of the transport equations are used to analyze crossover regimes and non-universal behavior. Orbital effects of a magnetic field are studied in section III. Large non-linear effects can be used as a tool to investigate the strongly anisotropic scattering from the spin waves. We will argue in section IV that these calculations easily explain some of the key observations in high purity single crystals close to an AFM QCP: the temperature dependence of the resistivity changes frap $\Delta \rho \propto T^{1.5}$ to $\Delta \rho \propto T$ for cleaner and cleaner systems 5 6. We propose a number of stringent tests for our picture and discuss how the orbital effects of a magnetic field can be separated from spin-contributions. We conclude in section $\mathrm{Z}$ by commenting on the relevance of this work to a wider class of problems.

\section{RESISTIVITY AT THE QCP}

\section{A. Model}

Following Hertzente 16 a spin-density antiferromagnetic transition in a metal can be modeled by an effective Ginzburg-Landau theory defined by the action

$$
S=\frac{1}{\beta} \sum_{\omega_{n}, \mathbf{q}} \Phi_{\mathbf{q}, i \omega_{n}}^{*}\left(r+\frac{(\mathbf{q} \pm \mathbf{Q})^{2}}{q_{0}^{2}}+\frac{\left|\omega_{n}\right|}{\Gamma}\right) \Phi_{\mathbf{q}, i \omega_{n}}
$$

$$
+U \int_{0}^{\beta} d \tau \int d^{3} \mathbf{r}|\Phi(\mathbf{r}, \tau)|^{4}
$$

where $\Phi$ is the order parameter which fluctuates in space and (imaginary) time, $\beta=1 / k_{B} T$ is the inverse temperature, $\omega_{n}=2 \pi n / \beta$ are bosonic Matsubara frequencies. The fluctuations are strongest near the ordering vector $\mathbf{Q}$ of the AFM. The mass $r$ controls the distance from the QCP. The Landau damping term linear in $|\omega|$ is due to the effective scattering of quasi-particles from spin-fluctuations. A large number of particle-hole pairs is created by a spin-fluctuation if $\mathbf{Q}$ connects different parts of the Fermi surface $\left(Q<2 k_{F}\right.$, see Fig-11). We will not discuss the singular case " $\mathbf{Q}=2 k_{F}, " 1,28$ where the Fermi velocities $\mathbf{v}_{\mathbf{k}}$ and $\mathbf{v}_{\mathbf{k} \pm \mathbf{Q}}$ are parallel or " $\mathbf{Q}>2 k_{F}$ " where the spin-fluctuations do not couple directly to the quasi particles.

Due to the ohmic Landau damping, the effective field theory (11) is characterized by a dynamic exponent $z=2$ $\left(\omega \sim q^{z}\right)$ and is for $d=3$ above its upper critical dimension 15 .16, $d+z>4$. Therefore the dynamic susceptibility is mainly determined by the gaussian part of (1) and the spin-fluctuation spectrum in the paramagnetic phase can be modeled by $15-1$

$$
\chi_{\mathbf{q}}(\omega)=\chi_{-\mathbf{q}}(\omega) \approx \frac{1}{1 /\left(q_{0} \xi\right)^{2}+\omega_{\mathbf{q}}-i \omega / \Gamma},
$$

where $q_{0} \approx k_{F}$ and $\Gamma$ are characteristic momentum and energy scales and $\xi$ is the correlation length of the spin fluctuations. The dispersion $\omega_{\mathbf{q}} \geq 0$ vanishes at the ordering wave vectors $\mathbf{Q}_{i}$ and varies on the scale $q_{0}$. For simplicity, we willmainly consider "isotropic" momentum dependences $29 \omega_{\mathbf{q}} \approx\left(\left(\mathbf{q} \pm \mathbf{Q}_{i}\right) / q_{0}\right)^{2}$ (appendix D defines the models used for numerical calculations more precisely). Moderate anisotropy influences our results only slightly, as will become clearer bolew. $\xi$ is the AFM correlation length which diverges 13,16 at the QCP as $1 / \xi^{2} \propto U T^{3 / 2}$. For the purposes of our numerical calculations we set

$$
1 /\left(q_{0} \xi\right)^{2}=r+c(T / \Gamma)^{3 / 2}
$$

with $c=1$. The $T$-dependence of $\xi$ does not affect the low-temperature properties (below Eqn. (28) a remarkable exception is discussed). We use $c=1$ to model the (non-universal) destruction of the spin fluctuations at the temperature scale $\Gamma$. The parameters in (2) can directly be obtained from inelastic neutron scattering. Typically, it is expected that in a heavy Fermion system, $\Gamma$ is of the order of the coherence-temperature or Kondo energy $T_{K}$. Close to the QCP, $r$ is a linear function of the tuning parameter, e.g. $r \propto p-p_{c}$ in a pressure tuned experiment.

Transport can be treated within a simple (quantum-) Boltzmann approach, because the spin-spin interactions are irrelevant in the renormalization group sense and because furthermore the concept of Fermi quasi particles is still valid 30 in 3 dimensions. For small and static electric fields the transport equations take the usual form 
of fermions scattering from bosonic excitations. These equations are further simplified by the assumption that the spin-fluctuations stay in equilibrium, i.e. we neglect drag effects. This approximation implicitly assumes the presence sufficient momentum relaxation e.g. by strong Umklapp scattering. In the linear response regime, the quasi particle distribution $f_{\mathbf{k}}=f_{\mathbf{k}}^{0}-\Phi_{\mathbf{k}}\left(\partial f_{\mathbf{k}}^{0} / \partial \epsilon_{\mathbf{k}}\right)$ is linearized around the Fermi distribution $f_{\mathbf{k}}^{0}$ and the collision term reads27

$$
\begin{aligned}
& \left.\frac{\partial f_{\mathbf{k}}}{\partial t}\right|_{\text {coll }}=\sum_{\mathbf{k}^{\prime}} \frac{f_{\mathbf{k}^{\prime}}^{0}\left(1-f_{\mathbf{k}}^{0}\right)}{T}\left(\Phi_{\mathbf{k}}-\Phi_{\mathbf{k}^{\prime}}\right) \\
& \times\left[g_{\mathrm{imp}}^{2} \delta\left(\epsilon_{\mathbf{k}}-\epsilon_{\mathbf{k}^{\prime}}\right)+\frac{2 g_{S}^{2}}{\Gamma} n_{\epsilon_{\mathbf{k}}-\epsilon_{\mathbf{k}^{\prime}}}^{0} \operatorname{Im} \chi_{\mathbf{k}-\mathbf{k}^{\prime}}\left(\epsilon_{\mathbf{k}}-\epsilon_{\mathbf{k}^{\prime}}\right)\right]
\end{aligned}
$$

Here $g_{\mathrm{imp}}^{2}$ and $g_{S}^{2}$ are transition rates for impurity scattering and inelastic scattering from spin fluctuations, respectively, and $n_{\omega}^{0}$ is the Bose function.
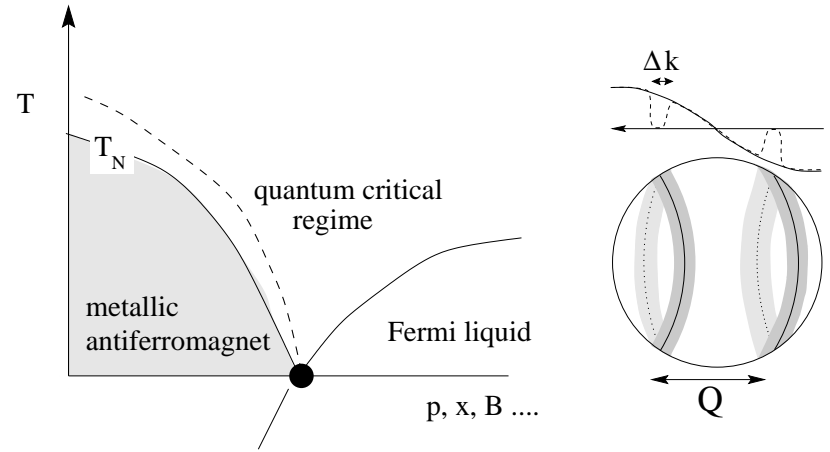

quantum critical point $(\mathrm{QCP})$

FIG. 1. Left figure: In a metallic AFM the magnetic order is suppressed as a function of some control parameter which can e.g. be pressure, doping or magnetic field. Deviations from the usual Fermi liquid behavior show up close to the QCP. Right figure: Near the transition to an antiferromagnet with ordering vector $\mathbf{Q}$, the scattering on the Fermi-surface is enhanced along "hot lines" connected by $\mathbf{Q}$. This strong scattering equilibrates the distribution function (shown for an electric field $\mathbf{E}$ parallel to $\mathbf{Q}$ ) in a region of width $\Delta k$ (see the main text and appendix A for details).

In the following we will show that the interplay of the two scattering mechanisms is highly non-trivial because of their completely different momentum dependence. On the one hand, impurity scattering is very isotropic and is equally efficient on the whole Fermi surface. Therefore, the distribution function in the presence of a small electric field $\mathbf{E}$ is smooth $\Phi_{\mathbf{k}} \propto \mathbf{v}_{\mathbf{k}} \mathbf{E}$ if impurity scattering dominates (solid line in the right part of Fig. 1). On the other hand the AFM susceptibility is strongly peaked around the ordering vectors $\mathbf{Q}_{i}$. Accordingly, a quasiparticle with an energy $\epsilon_{\mathbf{k}} \approx \mu$ will scatter efficiently from the spin fluctuations only near "hot lines" on the Fermi surface where $\epsilon_{\mathbf{k}_{H}} \approx \epsilon_{\mathbf{k}_{H} \pm \mathbf{Q}_{i}} \approx \mu$. This strong scattering tends to equilibrate the distribution function at points $\mathbf{k}_{H}$ and $\mathbf{k}_{H} \pm \mathbf{Q}_{i}$ in a region of width $\Delta k$ (dashed line in Fig. 1), where $\Delta k$ depends on the relative strength of impurity- and spin-fluctuation scattering. The temperature dependence of $\Delta k$ and the distortion of the distribution function close to the hot lines in a magnetic field is the main origin of the anomalous transport properties which are discussed in this paper (see appendix A for a simple qualitative calculation of $\Delta k$ ).

For convenience, we will use in the following the dimensionless quantities $t, x, r$ and $b$ to measure the effective temperature, the amount of disorder, the distance from the quantum-critical point and the strength of the magnetic field.

$$
\begin{gathered}
t=\frac{T}{\Gamma}, \quad x=\frac{\pi g_{\mathrm{imp}}^{2}}{2 g_{S}^{2}}=\frac{\rho_{0}}{\rho_{M}} \approx \frac{1}{\mathrm{RRR}}, \\
r=\frac{1}{\left(q_{0} \xi(t=0)\right)^{2}} \propto p-p_{c}, \quad b=\frac{B}{B_{0}}
\end{gathered}
$$

where $\rho_{0}=x \rho_{M}$ is the residual resistivity and $\rho_{M}$ is a typical high-temperature $(t \approx 1)$ resistivity which is defined below. One can approximately identify $x$ with the inverse of the residual resistivity ratio (RRR). $B_{0}$ is the typical magnetic field, which is necessary to see Shubnikov-de Haas oscillations at $t=1$

$$
\rho_{M}=\frac{3 \hbar g_{S}^{2}}{\pi e^{2} v_{F}^{2}}, \quad B_{0}=\frac{2 g_{S}^{2} q_{0}^{d}}{e v_{F}^{2}(2 \pi)^{d}}
$$

The Fermi velocity $v_{F}$ is defined by an average over the Fermi surface $v_{F}^{2} / 3=2 / N_{F} \iint d \mathbf{k}\left(\mathbf{v}_{\mathbf{k}} \mathbf{n}\right)^{2} /\left(v_{\mathbf{k}}(2 \pi)^{3}\right)$ where $\mathbf{n}$ is a unit vector in the direction of the electric field. $\iint d \mathbf{k} / v_{\mathbf{k}}=\int \delta\left(\epsilon_{\mathbf{k}}-\mu\right) d^{d} \mathbf{k}$ is an integral over the Fermi surface in $d$ dimensions and $N_{F}=2 \iint d \mathbf{k} /\left(v_{\mathbf{k}}(2 \pi)^{3}\right)$ the density of states.

\section{B. Boltzmann equation and scaling}

The linearized Boltzmann equations with the collision term (位) can be written in the following form

$\mathbf{v}_{\mathbf{k}} \mathbf{E}+\left(\mathbf{v}_{\mathbf{k}} \times \mathbf{B}\right) \partial_{\mathbf{k}} \Phi_{\mathbf{k}}=\iint F_{\mathbf{k k}^{\prime}}\left(\Phi_{\mathbf{k}}-\Phi_{\mathbf{k}^{\prime}}\right) \frac{d \mathbf{k}^{\prime}}{v_{\mathbf{k}^{\prime}}(2 \pi)^{3}}$

where an integration over directions perpendicular to the Fermi surface has already been performed and all $\mathbf{k}$-vectors and integrations are restricted to the Fermi surface $\iint d \mathbf{k} / v_{\mathbf{k}} \equiv \int d^{d} \mathbf{k} \delta\left(\epsilon_{\mathbf{k}}-\mu\right)$ where $d$ is the number of dimensions. Currents are calculated from $j_{i}=$ $\iint v_{i} \Phi_{\mathbf{k}} d \mathbf{k} /\left(v_{\mathbf{k}}(2 \pi)^{3}\right)$.

$F_{\mathbf{k k}^{\prime}}$ includes contribution both from elastic scattering from impurities and inelastic scattering from spin fluctuations with

$$
\begin{aligned}
F_{\mathbf{k k}^{\natural}} & =g_{\mathrm{imp}}^{2}+\frac{2 g_{S}^{2}}{\Gamma T} \int_{0}^{\infty} \omega n_{\omega}^{0}\left[n_{\omega}^{0}+1\right] \operatorname{Im} \chi_{\mathbf{k}-\mathbf{k}^{\natural}}(\omega) d \omega . \\
& =g_{\mathrm{imp}}^{2}+2 g_{S}^{2} I\left(r / t+\omega_{q} /\left(q_{0}^{2} t\right)\right) .
\end{aligned}
$$

$I(y)$ is defined by equation (8) and (2) and is asymptotically given by $I(y \rightarrow 0) \approx \pi /(2 y)$ and $I(y \rightarrow \infty) \approx$ $\pi^{2} /\left(3 y^{2}\right)$. 
For large temperatures and extremely large magnetic fields the solution of (7) depends on all details of the Fermi surface and only numerical solutions are possible. However, close to the quantum critical point the resistivity shows scaling behavior and depends only slightly on the geometry and the Fermi velocities along the hot lines. In the following we will derive an approximate analytic solution of the Boltzmann equation in this regime, the approximations used are exact in a well-defined limit of weak disorder, low temperature, weak coupling and small magnetic fields which will be specified below. Some of our qualitative results, especially in the absence of a magnetic field, can be derived by using a simple variational approach (appendix A) or even in a simple relaxation time approximation which we present in appendix B. The relaxation time approximation or, equivalently, the omission of vertex correction is completely wrong in certain geometries and leads to large errors especially for the Hall coefficient as explained in appendix B.

At $T=0$ the scattering is purely elastic and the quasiparticle distribution function is given by $\Phi_{\mathbf{k}}^{0}(\mathbf{B}) \equiv$ $\Phi_{\mathbf{k}}(B, T=0)$ with $\Phi_{\mathbf{k}}^{0}(0)=e \mathbf{E v}_{\mathbf{k}} /\left(g_{\mathrm{imp}}^{2} N_{F}\right)$. Our strategy is to keep track of the change of $\Phi_{\mathbf{k}}$ close to the hot lines, where the strong spin-fluctuations tend to equilibrate the distribution function at the points $\mathbf{k}$ and $\mathbf{k} \pm \mathbf{Q}_{i}$ (Fig. 1). For sufficiently small temperatures $t^{2}<x$ the distribution function will remain undisturbed in the "cold regions" far away from the hot lines where the inelastic scattering is of order $t^{2}$ and the elastic scattering dominates. It is therefore conveniept to write the distribution function in the following form 33

$$
\Phi_{\mathbf{k}} \approx\left(1-p_{\mathbf{k}}\right) \Phi_{\mathbf{k}}^{0}+p_{\mathbf{k}} \Phi_{\mathbf{k} \pm \mathbf{Q}_{i}}^{0} .
$$

For strong spin-scattering $p_{\mathbf{k}}$ approaches a value close to $1 / 2$ near the hot lines but vanishes further away. It is helpful to use a coordinate system, where vectors on the Fermi surface $\mathbf{k}=\mathbf{k}_{H}+\mathbf{k}_{\perp}$ are split up in a vector $\mathbf{k}_{H}$ on the hot line and a perpendicular vector $\mathbf{k}_{\perp} \perp \mathbf{k}_{H}, \mathbf{v}_{\mathbf{k}}$. After a rescaling of the momenta using $\mathbf{k}=\boldsymbol{\kappa} q_{0} \sqrt{t}$, in the limit $t<\sqrt{x}$ the Boltzmann equation takes the following form

$$
\begin{array}{r}
t^{(d-1) / 2} \int d \boldsymbol{\kappa}^{`}\left(p \boldsymbol{\kappa}+p_{\mathbf{k}_{H} \pm \mathbf{Q}+\boldsymbol{\kappa}^{\prime}}-1\right) I\left(r / t+M_{\boldsymbol{\kappa} \boldsymbol{\kappa}^{\prime}}\right) \\
+\sqrt{\alpha_{H}} c_{\mathbf{k}_{H}} x p \boldsymbol{\kappa}=c_{\mathbf{k}_{H}}^{\prime} \frac{b}{\sqrt{t}}\left(\hat{\mathbf{v}}_{\mathbf{k}} \times \hat{\mathbf{B}}\right) \partial \boldsymbol{\kappa} p \boldsymbol{\kappa}
\end{array}
$$

with $I(y)=\int_{0}^{\infty} d z z^{2} n^{0}(z)\left(1+n^{0}(z)\right) /\left(y^{2}+z^{2}\right), \boldsymbol{\kappa}^{\prime}=$ $\boldsymbol{\kappa}_{\|}^{\prime}+\boldsymbol{\kappa}_{\perp}^{\prime}, M_{\boldsymbol{\kappa} \boldsymbol{\kappa}^{\prime}}=\kappa_{\|}^{\prime 2}+\left(\boldsymbol{\kappa}_{\perp}^{\prime}-\boldsymbol{\kappa}_{\perp}\right)^{2}, c_{\mathbf{k}_{H}}=$ $(2 \pi)^{d} N_{F} \sqrt{v_{\mathbf{k}_{H}} v_{\mathbf{k}_{H} \pm \mathbf{Q}}} /\left(\pi q_{0}^{2}\right), c_{\mathbf{k}_{H}}^{\prime}=v_{\mathbf{k}_{H}} v_{\mathbf{k}_{H} \pm \mathbf{Q}} / v_{F}^{2}$ and 22 $\alpha_{H}=v_{\mathbf{k}+\mathbf{Q}} / v_{\mathbf{k}}$. $\hat{\mathbf{B}}$ and $\hat{\mathbf{v}}_{\mathbf{k}}$ are unit-vectors in the direction of $\mathbf{B}$ and $\mathbf{v}_{\mathbf{k}}$, respectively. The strength of the magnetic field is measured in units of a typical field $B_{0}$ defined in (5) and (6). One can think of $b$ as the typical angle by which an electron is deflected within a typical scattering time $\tau_{M}=(2 \pi)^{d} v_{F} /\left(2 g_{s}^{2} q_{0}^{d-1}\right)$. We are only considering magnetic fields with $b \ll x<1$ (i.e. $\omega_{c} \tau \ll 1$ ), where
Shubnikov-de-Haas oscillations are absent at low temperatures. In (10) we have neglected sub-leading contributions e.g. of the form $p_{\mathbf{k}} \partial_{\mathbf{k}} \Phi_{k}^{0}$, which are small in this regime because $p_{\mathbf{k}}$ varies stronger with momentum than $\Phi_{k}^{0}$ and all inelastic corrections come from small areas on the Fermi surface. This is not true in the ultra-clean limit $x \ll t^{2}$ which has to be covered separately (e.g. in section III A). For completeness we discuss some of our result in $d$ dimensions, however, we are mainly interested in three dimensions. Note that the Boltzmann equation approach presumably break down in $d=2$ as the "hot" electrons acquire a lifetime 34 质 $\sqrt{T}$, but it was nevertheless widely used in literature27 35. Qualitative features like the interplay of hot and cold regions might still be correctly described by our approach even in $d=2$.

From (10) it is clear that only the combinations $t^{(d-1) / 2} / x, t / r$ and $b /(x \sqrt{t})$ determine $p_{\boldsymbol{\kappa}}$. Accordingly, the resistivity obeys a scaling relation

$$
\frac{\Delta \rho_{i j}}{\rho_{M}} \approx t^{\frac{d}{2}} f_{i j}\left(\frac{t^{\frac{d-1}{2}}}{x}, \frac{r^{\frac{d-1}{2}}}{x}, \frac{b}{x \sqrt{t}}\right)
$$

for $t \ll \sqrt{x} \ll 1, r \ll 1$ and sufficiently small $b$, where $f$ depends smoothly on the details of the Fermi surface near the hot spots and the directions of magnetic and electric fields. $\rho_{M}$ is defined in Eqn. (6). The relation (11) becomes exact in the scaling limit

$$
t, x, b, r \rightarrow 0, \quad \frac{t^{(d-1) / 2}}{x}, \frac{r}{t}, \frac{b}{x \sqrt{t}} \rightarrow \text { const } .
$$

The scaling limit serves as a convenient regime for analytic calculations. An experimental verification of the predicted scaling would be the most precise test of the underlying models. We will calculate the scaling function $f$ in the following paragraphs in detail. However, for some applications, e.g. the Hall effect discussed in section III A, the deviations from scaling are important and are calculated from a full numerical solution of the Boltzmann equation (7). A reader interested only in the qualitative results can jump directly to section IV A, where a short overview of the asymptotic behavior of $f_{i i}$ is given (the off-diagonal components $f_{i \neq j}$ vanish in the scaling limit, see section III A where the Hall effect is discussed).

The two dimensional coupled integro-differential equations (10) can be considerably simplified by realizing, that $p_{\mathbf{k}}$ varies smoothly parallel to the hot lines but the kernel $I\left(\boldsymbol{\kappa}_{\|}^{\prime}\right)$ restricts the integrations to small values of $\boldsymbol{\kappa}_{\|}^{\prime}$. This allows us to replace $p_{\mathbf{k}_{H} \pm \mathbf{Q}+\boldsymbol{\kappa}^{\prime}} \equiv \tilde{p}_{\kappa_{\|}+\kappa_{\|,}^{\prime}, \kappa_{\perp}^{\prime}}$ by $\tilde{p}_{\kappa_{\|}, \kappa_{\perp}^{\prime}}$ in (10) and to perform the $\kappa_{\|}^{\prime}$ integration. For the same reason we can neglect the contribution proportional to $\partial_{\kappa_{\|}} p \boldsymbol{\kappa}$. All these approximations are valid in the scaling limit (12). It is therefore sufficient to solve a family of two coupled one-dimensional integral equations which depend parametrically on $\mathbf{k}_{H}$ :

$$
t^{(d-1) / 2} \int d \kappa^{\prime}\left(p_{\kappa}+\tilde{p}_{\kappa^{\prime}}-1\right) G_{\kappa \kappa^{\prime}}
$$




$$
\begin{aligned}
& +\sqrt{\alpha_{H}} c_{\mathbf{k}_{H}} x p_{\kappa}=c_{\mathbf{k}_{H}}^{\prime} \cos \theta_{B} \frac{b}{\sqrt{t}} \partial_{\kappa} p_{\mathbf{k}} \\
& t^{(d-1) / 2} \int d \kappa^{\prime}\left(\tilde{p}_{\kappa}+p_{\kappa^{\prime}}-1\right) G_{\kappa \kappa^{\prime}} \\
& +\sqrt{\frac{1}{\alpha_{H}}} c_{\mathbf{k}_{H}} x \tilde{p}_{\kappa}=c_{\mathbf{k}_{H}}^{\prime} \cos \theta_{B} \frac{b}{\sqrt{t}} \partial_{\kappa} \tilde{p}_{\mathbf{k}}
\end{aligned}
$$

where $\theta_{B}$ is the angle between $\mathbf{B}$ and a vector parallel to the hot lines at $\mathbf{k}_{H}$ and

$$
\begin{aligned}
G_{\kappa_{\perp} \kappa_{\perp}^{\prime}} & =\int d^{d-2} \mathbf{k}_{\|} I\left(r / t+M_{\boldsymbol{\kappa} \kappa^{\prime}}\right) \\
& \approx\left\{\begin{array}{ll}
\frac{1}{2 \sqrt{a_{\kappa \kappa^{\prime}}}} & a_{\kappa \kappa^{\prime}} \ll 1 \\
\frac{\pi}{6 a_{\kappa \kappa^{\prime}}^{3 / 2}} & a_{\kappa \kappa^{\prime}} \gg 1
\end{array} \text { for } d=3 .\right.
\end{aligned}
$$

with $a_{\kappa \kappa^{\prime}}=r / t+\kappa^{2}+\kappa^{\prime 2}-2 \kappa \kappa^{\prime} \cos \phi$, where $\phi \neq 0$ is the angle between $\mathbf{v}_{\mathbf{k}_{H}}$ and $\mathbf{v}_{\mathbf{k}_{H} \pm \mathbf{Q}}$. The equation for $\tilde{p}_{\kappa}$ is obtained by replacing $\alpha_{H}$ by $1 / \alpha_{H}$ and exchanging $p_{\kappa}$ and $\tilde{p}_{\kappa}$ in (13). The boundary condition for (13) are $p_{\kappa}, \tilde{p}_{\kappa} \rightarrow 0$ for $\kappa \rightarrow \pm \infty$ by construction.

Currents are calculated from

$$
\begin{aligned}
j^{i}= & j_{0}^{i}+ \\
& 2 \sum_{i} \oint_{i} \frac{d \mathbf{k}_{H}}{v_{\mathbf{k}_{H}}(2 \pi)^{3}} v_{\mathbf{k}_{H}}^{i}\left(\Phi_{\mathbf{k}_{H}}^{0}-\Phi_{\mathbf{k}_{H}+\mathbf{Q}}^{0}\right) q_{0} \sqrt{t} \int d \kappa p_{\kappa}
\end{aligned}
$$

where $\mathbf{j}_{0}=2 \iint \mathbf{v}_{\mathbf{k}} \Phi_{\mathbf{k}_{H}}^{0} d^{3} \mathbf{k} /\left(v_{\mathbf{k}}(2 \pi)^{3}\right)$ is the current in the absence of inelastic scattering, $\oint_{i} d \mathbf{k}_{\|}$denotes the line integral along the $i$ th hot line and the expression is summed over all hot lines $i$. Note that $p_{\kappa}$ is a function of $\mathbf{k}_{H}$. In the scaling limit $b \ll x$, the magnetic field dependence of $\Phi_{\mathbf{k}_{H}}^{0} \propto \mathbf{v}_{\mathbf{k}_{\mathbf{H}}} \mathbf{E}$ can be neglected in the second term in (16). Using the symmetries of the Boltzmann equations one can derive the convenient expression

$$
\frac{\Delta \rho_{i j}}{\rho_{M}} \approx x \frac{\sum_{i} \oint_{i} \frac{d \mathbf{k}_{\|}}{v_{\mathbf{k}_{H}}} \Delta v^{i} \Delta v^{j}\left(q_{0} \sqrt{t}\right) \int d \kappa p_{\kappa}}{\int\left(v_{\mathbf{k}}^{i}\right)^{2} \frac{d \mathbf{k}_{\|}}{v_{\mathbf{k}}}} .
$$

with $\Delta v^{i}=v_{\mathbf{k}_{H}}^{i}-v_{\mathbf{k}_{H} \pm \mathbf{Q}}^{i}$.

\section{Transport for $B=0$}

In the absence of a magnetic field, two different regimes emerge depending on whether the elastic scattering dominates close to the hot line25,26. If impurity scattering dominates $(t<\min [x, \sqrt{r x}])$, it smears out the quasi particle distribution ( $\Delta k \approx 0$ in Fig. 11). $p_{\kappa}$ in (13) is small and in leading order in $p_{\kappa}$ one obtains

$$
\begin{aligned}
\frac{\Delta \rho}{\rho_{M}} \approx & t^{3 / 2} h\left(\frac{t}{r}\right) \frac{3 q_{0}^{3}}{8 \pi^{4}\left(v_{F} N_{F}\right)^{2}} \\
& \times \sum_{i} \oint_{i} d \mathbf{k}_{\|} \frac{\left(\left(\mathbf{v}_{\mathbf{k}_{\|}}-\mathbf{v}_{\mathbf{k}_{\|} \pm \mathbf{Q}_{i}}\right) \mathbf{n}\right)^{2}}{\left|\mathbf{v}_{\mathbf{k}_{\|}} \times \mathbf{v}_{\mathbf{k}_{\|} \pm \mathbf{Q}_{i}}\right|}
\end{aligned}
$$

$$
\begin{aligned}
h\left(\frac{t}{r}\right) & =\frac{\pi}{2} \int_{0}^{\infty} y n^{0}(y)\left(1+n^{0}(y)\right) \operatorname{Im} \sqrt{\frac{r}{t}+i y} \\
& \approx \begin{cases}\frac{\pi \zeta(3 / 2) \Gamma(5 / 2)}{2 \sqrt{2}} & , t>r \\
\frac{\pi^{3}}{12} \sqrt{t / r} & , t<r\end{cases}
\end{aligned}
$$

In this dirty limit, we recover the well-known $T^{1.5}$ resistivity at the QCP. This result is usually_derived by an average over the inelastic scattering rate 17 . We want to stress that this approximation can be applied only in the presence of strong impurity scattering and gives wrong results for cleaner systems.

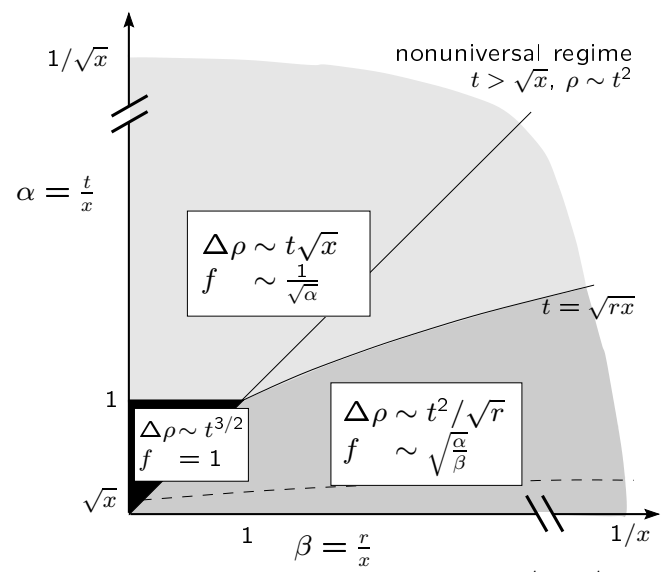

FIG. 2. In the scaling limit $t, x, r \rightarrow 0, t / x, r / x \rightarrow$ const. the resistivity is "universal" $\Delta \rho / \rho_{M}=t^{3 / 2} f(t / x, r / x)$ for $t<\sqrt{x}, r<1$, where $t \propto T, x \propto \rho_{0}$ and $r \propto p-p_{c}$ measure the temperature, the amount of disorder and the distance from the QCP. The plot shows the qualitative behavior of the scaling function $f[\alpha, \beta]$ in the various regimes. For $\min [x, \sqrt{r x}<t<\sqrt{x}]$ the resistivity rises linearly with temperature. Thermodynamic quantities show a crossover to Fermi liquid behavior at the scale $t=r$ (straight line), while in transport a $T^{2}$ behavior is recovered at a much smaller scale $\min [x, \sqrt{r x}]$. The dashed line serves as a reminder that at lowest temperatures effects which are not included in our approach become impertant, e.g. interference effects of disorder and interaction $\$ 36$ pra disorder induced change of the spin fluctuation spectrum 24 .

To derive (18) we considered for simplicity isotropic spin fluctuations. In the case of a moderate anisotropy our results have to be changed slightly. For example, if the anisotropy of the spin fluctuations is described by $\mathbf{q}_{i} g_{i j} \mathbf{q}_{j} / q_{0}^{2}$, then the denominator of the integral in (18) has to be replaced by $v_{\mathbf{k}_{H}} v_{\mathbf{k}_{H} \pm \mathbf{Q}} \operatorname{det}^{1 / 2} \sum_{i j} \hat{\mathbf{k}}_{i}^{\alpha} g_{i j} \hat{\mathbf{k}}_{j}^{\beta}$ where the $\mathbf{k}^{1}$ is a unit vector parallel to the hot line and $\mathbf{k}^{2}, \mathbf{k}^{3}$ are unit vectors perpendicular to $\mathbf{k}^{1}$ and either $\mathbf{v}_{\mathbf{k}_{H}}$ or $\mathbf{v}_{\mathbf{k}_{H} \pm \mathbf{Q}}$. The determinant is always finite because we do not consider the case $\mathbf{v}_{\mathbf{k}_{\|}} \| \mathbf{v}_{\mathbf{k}_{\|}+\mathbf{Q}}\left(" \mathbf{Q}=2 k_{F}\right.$ "). 


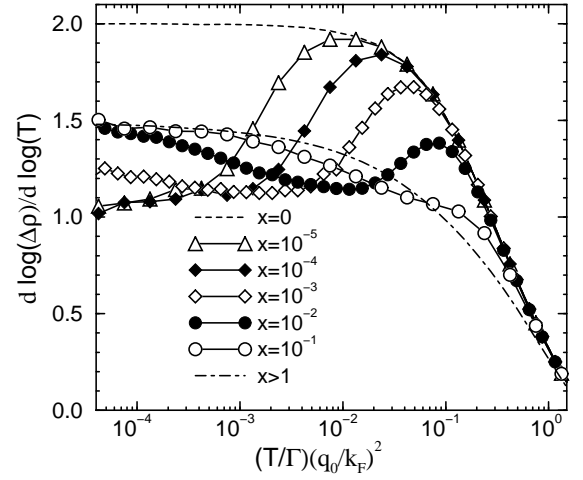

FIG. 3. Effective exponent of the resistivity at the QCP $(r=0)$, defined as the logarithmic derivative of $\Delta \rho(T)$. At very low temperatures, the "dirty-limit" exponent $3 / 2$ is recovered. However, in the experimentally accessible low temperature regime smaller exponents are to be expected for rather clean system $(x<0.1)$.

In the limit $\sqrt{x}>t>\min [x, \sqrt{r x}]$, the resistivity rises linearly with temperature. The origin of this effect is that the spin fluctuations equilibrate a region of width $\Delta k \approx t / \sqrt{x}$ (Fig. 1) as we will show in Eqn. (29) (or appendix A). Therefore we obtain $\rho \sim x(1+t / \sqrt{x})$ or more precisely

$$
\begin{aligned}
\frac{\Delta \rho}{\rho_{M}} \approx & t \sqrt{x}\left[\frac{q_{0}^{2} \sqrt{\pi / 3}}{2 v_{F}^{2}(2 \pi)^{3} N_{F}^{3 / 2}} \sum_{i} \oint_{i} d \mathbf{k}_{\|}[\right. \\
& \left.\left.\frac{\left(\left(\mathbf{v}_{\mathbf{k}_{\|}}-\mathbf{v}_{\mathbf{k}_{\|} \pm \mathbf{Q}_{i}}\right) \mathbf{n}\right)^{2}}{\left|\mathbf{v}_{\mathbf{k}_{\|}} \times \mathbf{v}_{\mathbf{k}_{\|} \pm \mathbf{Q}_{i}}\right|} \frac{\mathbf{v}_{\mathbf{k}_{\|} \pm \mathbf{Q}_{i}}^{5 / 4}}{\mathbf{v}_{\mathbf{k}_{\|}}^{3 / 4}} S_{\mathbf{v}_{\mathbf{k}_{\|} \pm \mathbf{Q}_{i}} / \hat{\mathbf{v}}_{\mathbf{k}_{\|} \pm \mathbf{v}_{\|}} \hat{\mathbf{v}}_{\|} \mid}\right]\right]
\end{aligned}
$$

where the only slightly varying function $S_{\alpha}^{a}=$ $\int_{-\infty}^{\infty} d \kappa p_{\kappa}(a, \alpha) \approx \pi$ is calculated from the solution of the two coupled one-dimensional integral equations (see Eqn. (29))

$$
\begin{aligned}
& p_{q}+\int d q^{\prime} \frac{\left(p_{q}+\tilde{p}_{q^{\prime}}-1\right)\left(1-a^{2}\right)}{\left(q^{2} / \alpha+q^{\prime 2} \alpha-2 q q^{\prime} a\right)^{3 / 2}}=0 \\
& \tilde{p}_{q}+\int d q^{\prime} \frac{\left(\tilde{p}_{q}+p_{q^{\prime}}-1\right)\left(1-a^{2}\right)}{\left(q^{2} \alpha+q^{\prime 2} / \alpha-2 q q^{\prime} a\right)^{3 / 2}}=0
\end{aligned}
$$

which in the limit $a \rightarrow \pm 1$ are solved by $p_{q}=1 /((1+$ $\alpha)+q^{2} / 2 \sqrt{\alpha}$ ) (see Fig. 8).

Omitting all prefactors we obtain in the scaling regime $r \ll 1, t<\sqrt{x}$

$$
\begin{aligned}
\frac{\Delta \rho}{\rho_{M}} & \approx t^{\frac{d}{2}} f\left(\frac{t^{\frac{d-1}{2}}}{x}, \frac{r^{\frac{d-1}{2}}}{x}\right) \\
& \sim \begin{cases}t^{d / 2} & , r<t<x^{2 / d-1} \\
t^{\frac{2}{5-d}} x^{\frac{4-d}{5-d}} & , \max \left[x^{\frac{2}{d-1}}, \sqrt{x} r^{\frac{5-d}{4}}\right]<t<\sqrt{x} \\
t^{2} / r^{2-\frac{d}{2}} & , t<\min \left[r, \sqrt{x} r^{\frac{5-d}{4}}\right]\end{cases}
\end{aligned}
$$

where for completeness we have given the result in arbitrary dimensions $d$ (see appendix A for a simple derivation of these results). A summary of the scaling behavior in $d=3$ is shown in Fig. 2. It is worthwhile to point out, that even away from the QCP, for $r>0$, there is a large regime with a NFL resistivity (Fig. 1 and 5 ). To actually observe the $T^{2}$-term one has to consider very low temperatures $t<\sqrt{r x}$. This has to be compared to thermodynamic quantities, where the crossover to Fermiliquid behavior is expected at a higher scale $t \sim r$. In a very clean system these scales can be quite different and anomalous transport should be observed in regimes where thermodynamic quantities display typical Fermi liquid properties.

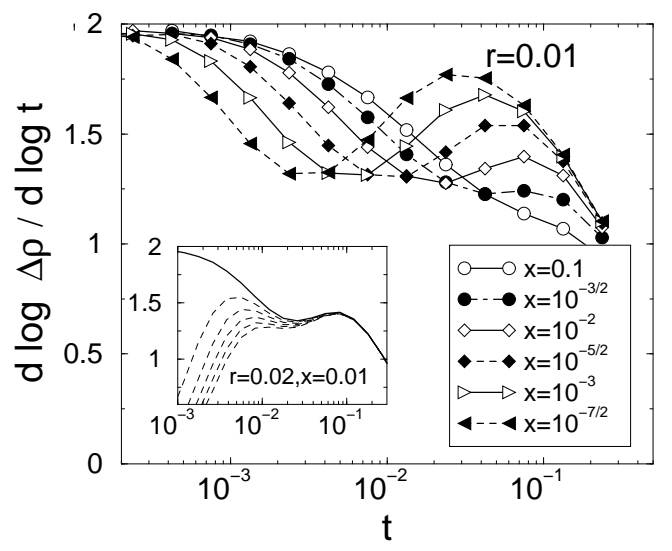

FIG. 4. Effective exponent, defined as the logarithmic derivative of $\Delta \rho(T)$, for a fixed distance from the QCP $r=0.01$ and various values of disorder. In high purity samples the crossover to an exponent 2 can be seen only at lowest temperature $t \ll \min [x, \sqrt{r x}, r]$ (18). The bump at higher temperatures is a precursor of the effect that in ultra clean samples, $x \ll t^{2} \ll 1$, we expect $\Delta \rho \sim t^{2}$ (22). In the scaling limit (12) the resistivity is linear in the intermediate regime $\sqrt{r x}<t<\sqrt{x}(20)$. For the chosen parameters this exponent 1 cannot be identified, however, a pronounced regime with an effective exponent less than 1.5 is seen The inset illustrates the problem to extract the exponent 68 from the logarithmic derivative of $\Delta \rho=\rho-\rho_{0}$ if $\rho_{0}$ is not known. For $=0.02$ and $x=0.01$ the logarithmic derivative of $\rho-(1-\epsilon) \rho_{0}$ for $\epsilon=2-10 \%$ (dashed lines) is compared to the "true" exponent for $\epsilon=0$ (solid line).

For higher temperatures $t>\sqrt{x}$, larger resistivities $\Delta \rho \gg \rho_{0}$ or further away from the QCP $r \sim 1$ the resistivity is much less universal and one has to rely on numerical solutions of the Boltzmann equation. In the ultra-clean limit $x<t^{2} \ll 1$, the distribution function $\Phi_{\mathbf{k}}$ is suppressed in a large region around the hot lines 27 $\left(\Delta k \approx O\left(k_{F}\right)\right.$ in Fig. 1] $)$. Therefore only non-singular scattering dominates the resistivity and we obtain

$$
\frac{\rho}{\rho_{M}} \approx t^{2}
$$

with a prefactor which to leading order is independent of the distance from the QCP $r$ but depends on all details of the Fermi surface and of the interactions far from the hot lines. Hlubina and Rice27 were the first to discuss 
this regime where quantum-critical effects cannot be seen in the transport because the long-living quasi particles in the "cold regions" short-circuit all contributions from the hot lines. Our numerical solutions, however, suggest that this regime is experimentally not accessible. Fig. 3 shows the logarithmic derivative of $\Delta \rho$, which defines an effective exponent $\alpha, \Delta \rho \propto T^{\alpha}$. Even for an extremely clean system with a residual resistivity ratio $(R R R \approx$ $1 / x)$ of the order of $10^{4}$, there is never an extended range of temperatures, where this effective exponent is close to 2. On the other hand we see that in moderately clean systems with $x<0.1$ there is always a regime, where the effective exponent is smaller than 1.5. Sufficiently clean systems clearly show the linear resistivity predicted by (20) over a large range.

The full scaling function for $r=0$ and the deviations from scaling are shown in Fig. 11 .

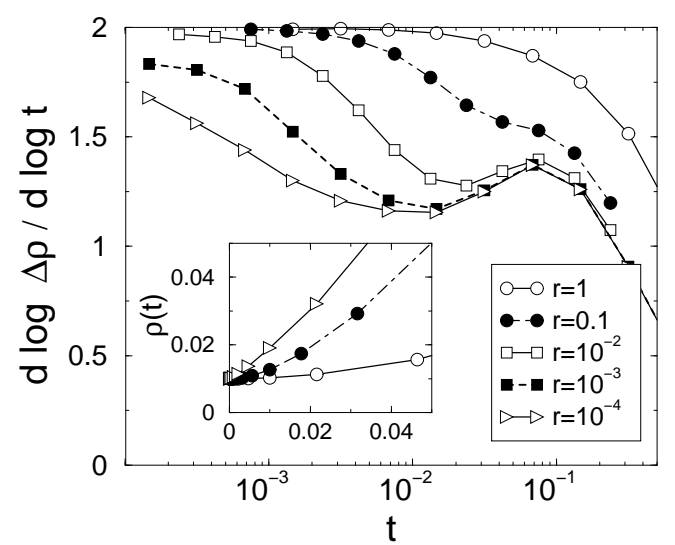

FIG. 5. Effective exponent, defined as the logarithmic derivative of $\Delta \rho(T)$, for a relatively clean sample with $x=0.01$ and various values of $r \propto p-p_{c}$. The resistivity is calculated from a numerical solution of the Boltzmann equation for model A defined in appendix D. The inset shows $\rho(t)$ for $r=0,0.1,1$.

\section{MAGNETOTRANSPORT}

The characteristic feature of the spin-density wave scenario is, that only the quasi particles close to "hot lines" are strongly affected by the spin-fluctuations. This has to be contrasted with a situation where the Kondo effect is destroyed close to the QCP which would affect the full Fermi surface. It is therefore important to have a probe for testing the presence of sharp structures on the Fermi surface.

The (nonlinear) magnetoresistivity can be used to investigate precisely this question. By a magnetic field, the quasi particles are driven parallel to the Fermi surface by the force $\mathbf{v}_{\mathbf{k}} \times \mathbf{B}$. Generally, non-linear effects in the magnetotransport show up if the quasi particles are able to circle the Fermi surface without being scattered, i.e. for $\omega_{c} \tau \gtrsim 1$, where $\omega_{c}$ is the cyclotron frequency. However, if there is a small region of width $\Delta k$ on the
Fermi surface where the scattering is relatively strong (or weak) then non-linear effects will become important for $\omega_{c} \tau \gtrsim \Delta k / k_{F}$. Close the AFM quantum critical point the width of hot lines $\Delta k$ vanishes in the low-temperature limit and the quasi particles can be driven by a moderate magnetic field over the hot lines. This will lead to large effects in the magnetotransport. The magnetotransport in nearly antiferromasnetic two dimensional metals has been studied before 35 , 50 in detail in the context of hightemperature superconductors. However, the authors did not focus on the role of weak disorder, which we find at least in three dimensions to be essential to describe the low-temperature the magnetotransport both quantitatively and qualitatively.

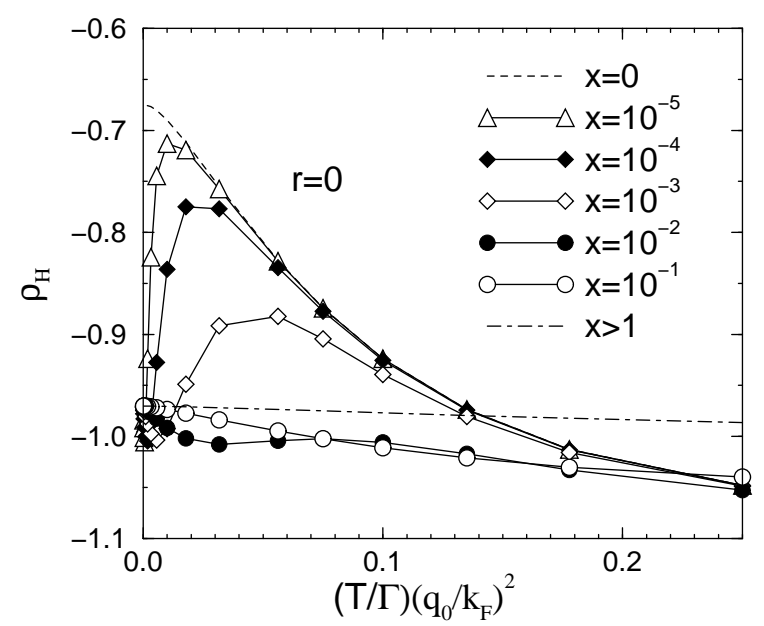

FIG. 6. Hall effect for model A, calculated from a numerical solution of 7. Extremely small amounts of disorder strongly influence the Hall effect. Very clean samples are necessary to observe this effect. Both the sign and the size of the effect depend on details of the band structure.

\section{A. Hall effect}

The Hall effect is extremely sensitive to small amounts of disorder $\left(x<t^{2}\right)$ but essentially constant for $t \ll \sqrt{x}$.

This can be seen most easily from the symmetry properties of the Boltzmann equation (13) in the scaling limit. The left-hand side of (13) is even in $\kappa$, while the right hand side is odd and linear in the magnetic field. Therefore $\int_{-\infty}^{\infty} p_{\kappa}$ is even in $\mathbf{B}$ and there is no contribution to the Hall effect from the hot lines in the scaling limit (12) where (13) and (17) are valid. This does not imply that the Hall effect is unaffected by the singular scattering close to the QCP. It is on the contrary an extremely sensitive probe to very small amounts of impurity scattering. The Hall constant at $T=0$ is different in the ultra-clean and the dirty limit $R_{H}\left(t \rightarrow 0, x \ll t^{2}\right) \neq R_{H}(t \rightarrow 0, x=$ const.). In the former limit the elastic impurity scattering dominates in the latter the magnetic scattering. The quasiparticle distribution $\Phi_{\mathbf{k}}$ has a completely different momentum dependence in these two limits. One 
consequence is that a different average over the effective masses enter the Hall constant. Small amounts of disorder $x \sim t^{2}$ change the distribution $\Phi_{\mathbf{k}}$ completely and give rise to the strong features in the Hall effect shown in Fig. 6. The calculation is based on a numerical solution of the full Boltzmann equations (7) for small magnetic fields. Even disorder at the level of e.g. $x=10^{-4}$ leads to a strong structure in the Hall effect at quite high temperatures. Extremely clean samples are necessary to observe the strong temperature dependence of $R_{H}$. Both the sign and the size of these effects are non-universal and depend on the details of the band structure.

\section{B. Magnetoresistivity - small fields}

While all corrections to the Hall effect cancel in the scaling limit (12), the magnetoresistivity is strongly influenced by the hot lines and can be used to investigate the quantum-critical properties in more detail.

We first discuss the dirty limit $x>t(d=3)$. $p_{\kappa}$ is small in this case allowing an expansion of (13) in $p_{\kappa}$. In order $b^{2}$, the distribution function changes by $\Delta p_{\kappa} \approx\left(b \cos \Theta_{B} c_{\mathbf{k}_{H}}^{\prime} /\left(x \sqrt{t} c_{\mathbf{k}_{H}} \sqrt{\alpha_{H}}\right)\right)^{2} \partial_{\kappa}^{2} p_{\kappa}^{0}$ where $p_{\kappa}^{0}$ is the distribution function for $b=0$. The resistivity (17) is proportional to $\int p_{\kappa}$, but $\int \Delta p_{\kappa}$ vanishes obviously. Therefore it is useful to integrate (13)

$$
\int_{-\infty}^{\infty} d \kappa p_{\kappa}=\frac{t}{x \sqrt{\alpha_{H}} c_{\mathbf{k}_{H}}} \iint_{-\infty}^{\infty} d \kappa d \kappa^{\prime} G_{\kappa \kappa^{\prime}}\left(1-p_{\kappa}-\tilde{p}_{\kappa^{\prime}}\right) .
$$

We calculate the leading correction to $\int p_{\kappa}$ from the right side of (23), using that $p_{\kappa}^{0} \approx t /\left(x \sqrt{\alpha_{H}} c_{\mathbf{k}_{H}}\right) \int G_{\kappa \kappa^{\prime}} d \kappa^{\prime}$ :

$$
\Delta \int p_{\kappa} \approx \frac{1+\alpha_{H}^{3}}{\alpha_{H}}\left(\frac{b \cos \Theta_{B} c_{\mathbf{k}_{H}}^{\prime}}{x \sqrt{t} c_{\mathbf{k}_{H}}}\right)^{2} \int d \kappa\left(\partial_{\kappa} p_{\kappa}^{0}\right)^{2}
$$

and obtain for $r<t<\min [x, \sqrt{r x}], b \ll x \sqrt{r}$

$$
\begin{aligned}
\frac{\Delta \rho}{\rho_{M}} \approx & b^{2} \frac{t^{4}}{x^{3} r^{5 / 2}}\left[\frac { \pi ^ { 7 } q _ { 0 } ^ { 9 } / ( 1 2 v _ { F } ^ { 4 } ) } { ( N _ { F } ( 2 \pi ) ^ { 3 } ) ^ { 5 } } \sum _ { i } \oint _ { i } d \mathbf { k } _ { \| } \left[\frac{v_{\mathbf{k}_{\|}}^{3}+v_{\mathbf{k}_{\|} \pm \mathbf{Q}_{i}}^{3}}{v_{\mathbf{k}_{\|}}^{4} v_{\mathbf{k}_{\|} \pm \mathbf{Q}_{i}}^{4}}\right.\right. \\
& \left.\left.\frac{\left(\left(\mathbf{v}_{\mathbf{k}_{\|}}-\mathbf{v}_{\mathbf{k}_{\|} \pm \mathbf{Q}_{i}}\right) \mathbf{n}\right)^{2}\left(\left(\mathbf{v}_{\mathbf{k}_{\|}} \times \mathbf{v}_{\mathbf{k}_{\|} \pm \mathbf{Q}_{i}}\right) \hat{\mathbf{B}}\right)^{2}}{\left|\mathbf{v}_{\mathbf{k}_{\|}} \times \mathbf{v}_{\mathbf{k}_{\|} \pm \mathbf{Q}_{i}}\right|}\right]\right]
\end{aligned}
$$

where $\hat{\mathbf{B}}$ is a unit vector in the direction of the magnetic field. It is surprising that the leading low temperature correction in this Fermi liquid regime $t \ll r$ is not proportional to $T^{2}$ but starts with $T^{4}$. This is due to the above mentioned cancellations and is actually valid only for $t>r \sqrt{x}$ as shown below.

There are a number of other contributions to the magnetoresistivity which vanish in the scaling limit but might be dominating in the experimentally relevant regime. One important correction is due to the suppression of the AFM in a magnetic fields, we will discuss these effects in detail in section IVC. Before analyzing the magnetoresistivity it is usefull to subtract the $B$-dependence of the resual resistivity $\Delta \rho_{0} \sim b^{2} / x$ which is the largest correction to our results. Therefore we use in this section the definition

$$
\Delta \rho=(\rho(b, t)-\rho(0, t))-(\rho(b, 0)-\rho(0,0))
$$

Other sub-leading contributions become important for higher magnetic fields or in the case of Eqn. (25) for low temperatures. They are missing in (10) because we neglected terms of the form $\partial_{\mathbf{k}}\left(\Phi_{\mathbf{k}_{H}}^{0}-\Phi_{\mathbf{k}_{H} \pm \mathbf{Q}}^{0}\right)$. We estimate these corrections in the regime $\omega_{c} \tau \ll 1$ and $\Delta \rho<\rho_{0}$ using Kohler's rule $(\rho(T, B)-\rho(0, B) \propto$ $\left.B^{2} \Delta \rho(T, 0) / \rho(0,0)^{2}\right)$. In the regime discussed in (25), they are of the order of $\Delta \rho \propto b^{2} t^{2} /\left(\sqrt{r} x^{2}\right)$ and therefore (25) is valid only for $t>t^{*}=r \sqrt{x}$. In the scaling limit (12), $t^{*}$ vanishes.

The behavior in the disorder dominated quantumcritical regime $t<x, r \ll t$ is more complicated. For small momenta, $G_{\kappa \kappa^{\prime}}$ is approximated by $G_{\kappa \kappa^{\prime}} \approx$ $1 /\left(2 \sqrt{r / t+\kappa^{2}+\kappa^{\prime 2}-2 \kappa \kappa^{\prime} \cos \phi}\right)$, where $\phi$ is the angle between $\mathbf{v}_{\mathbf{k}_{H}}$ and $\mathbf{v}_{\mathbf{k}_{H}+\mathbf{Q}}$. In leading order in $t / x$ for $b=0$ and $\kappa<1$ Eqn. 7 is solved by

$$
p_{\kappa}^{0} \sim t / x \int G_{\kappa \kappa^{\prime}} d \kappa^{\prime} \sim t /(2 x) \ln \left[1 /\left(r / t+\kappa^{2}\right)\right] .
$$

where the prefactors and $\phi$ dependence in the argument of the log have been omitted. The approximations leading to (27) are valid for $p_{\kappa}^{0} \ll 1$, therefore they break down in an exponentially small regime $r / t+\kappa^{2} \lesssim$ $c e^{-x /(2 t)}$, where $c$ is a constant of order 1 .

With all prefactors, which are calculated using (24), for $e^{-x /(2 t)} \ll r<t<x$, the inelastic contribution to the magnetoresistivity is given by

$$
\begin{aligned}
& \frac{\Delta \rho}{\rho_{M}} \approx b^{2} \frac{t^{2}}{x^{3} \sqrt{r}}\left[\frac { 3 \pi ^ { 5 } q _ { 0 } ^ { 9 } / ( 2 v _ { F } ^ { 4 } ) } { ( N _ { F } ( 2 \pi ) ^ { 3 } ) ^ { 5 } } \sum _ { i } \oint _ { i } d \mathbf { k } _ { \| } \left[\frac{v_{\mathbf{k}_{\|}}^{3}+v_{\mathbf{k}_{\|} \pm \mathbf{Q}_{i}}^{3}}{v_{\mathbf{k}_{\|}}^{4} v_{\mathbf{k}_{\|} \pm \mathbf{Q}_{i}}^{4}}\right.\right.
\end{aligned}
$$

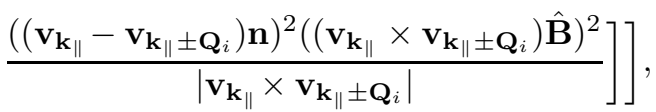

where the geometrical factors are accidentally exactly the same as in (25). It is quite surprising, that even in the limit $r \ll t$ the quadratic magnetoresistivity diverges proportional to $1 / \sqrt{r}$. What happens if a system is directly at the QCP, i.e. in the case $r=0$ ? In the scaling limit (12), the cutoff $r / t$ in (27) is replaced by $e^{-x /(2 t)}$ and the magnetoresistivity is proportional to $b^{2} t^{1.5} e^{x /(4 t)} / x^{3}$. This exponentially large correction is probably not experimentally observable. Either higher-order effects in the magnetic field will become relevant (to be discussed in the next section) or one has to take into account the temperature dependence of $r$. The low-temperature correction 16 $\Delta r \approx c^{\prime} t^{3 / 2}$ can formally be neglected in the scaling limit and usually leads only to small sub-leading corrections of the order of $\Delta r / t \approx c^{\prime} \sqrt{t}$ both in transport and thermodynamic quantities. The prefactor $c^{\prime}$ is proportional to 
the spin-spin interaction $U$ (11). The magnetoresistivity at the QCP in the dirty limit is a remarkable exception of this rule: for $r \ll t \ll x$ and $c^{\prime} \sqrt{t}>e^{-x /(2 t)}$ the temperature dependence of $r$ determines the $B^{2}$ correction to the resistivity and we expect $\Delta \rho \sim b^{2} t^{1.25} /\left(\sqrt{c^{\prime}} x^{3}\right)$ (28) ( see Fig. 16). An experimental confirmation of this prediction would be an interesting way to investigate the temperature dependence of $1 / \xi^{2}$, a quantity which can otherwise be measured only by high-precision inelastic neutron scattering.

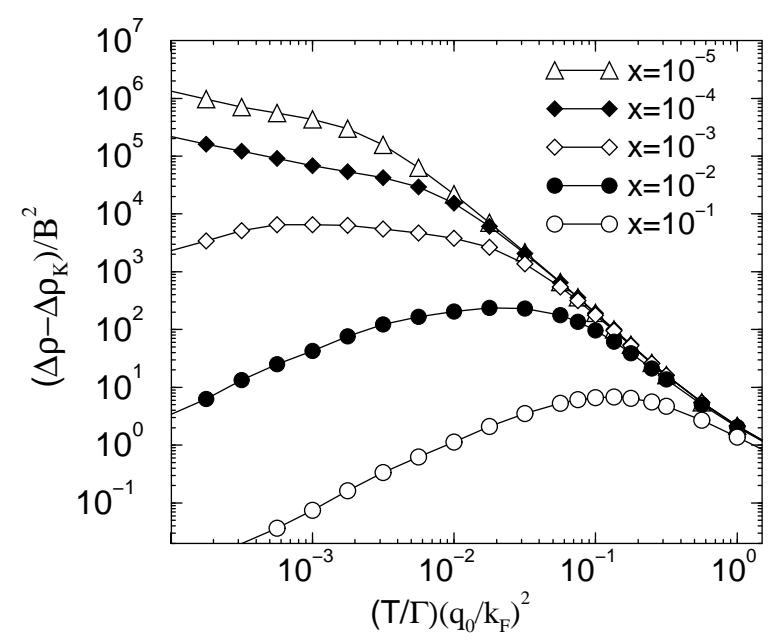

FIG. 7. Log-log plot of the temperature dependence of the magnetoresistivity $\Delta \rho / b^{2}-\Delta \rho_{K} / b^{2}$ at the QCP in a small magnetic field $b$ for model $A$, calculated from a numerical solution of (7). The "Kohler's rule"-contribution $\Delta \rho_{K}=\Delta \rho(t=0, b)(\rho(t=0, b=0) / \rho(t, b=0))$ has been subtracted. In the dirty limit, $t<x$, one can indentify the correction (28) with $\Delta \rho \sim b^{2} t^{2} /\left(x^{3} \sqrt{r}\right) \sim b^{2} t^{5 / 4} / x^{3}, r \approx t^{3 / 2}$ at the QCP. The magnetoresistivity in the clean regime, $x<t<\sqrt{x}$ is much larger with $\Delta \rho \sim b^{2} /(\sqrt{x} t)$ (see Eqn. (30)). In the ultra-clean limit, $x<t^{2}$, we obtain $\Delta \rho \sim 1 / t^{2}$ and to leading order no effects of the QCP can be seen.

The solution of the Boltzmann equation (13) in the regime $\max [x, \sqrt{r x}] \ll t<\sqrt{x}$ is dominated by large momenta $\kappa, \kappa^{\prime} \gg \max [1, \sqrt{r / t}]$. We therefore use the asymptotic form of $G_{\kappa \kappa^{\prime}}(15)$ and rescale the argument of $p_{\kappa}$ by $\kappa=q \sqrt{\pi t /\left(6 x c_{\mathbf{k}_{H}} \sin ^{2} \phi\right)} / \sqrt{\alpha_{H}}$ and the argument of $\tilde{p}_{\kappa^{\prime}}$ by $\kappa^{\prime}=q^{\prime} \sqrt{\pi t /\left(6 x c_{\mathbf{k}_{H}} \sin ^{2} \phi\right)} \sqrt{\alpha_{H}}$.

$p_{q}+\int \frac{d q^{\prime}\left(p_{q}+\tilde{p}_{q^{\prime}}-1\right) \sin ^{2} \phi}{\left(q^{2} / \alpha_{H}+q^{\prime 2} \alpha_{H}-2 q q^{\prime} \cos \phi\right)^{3 / 2}}=\frac{b \tilde{c}_{\mathbf{k}_{H}}}{t \sqrt{x}} \partial_{q} p_{q}$

$\tilde{p}_{q}+\int \frac{d q^{\prime}\left(\tilde{p}_{q}+p_{q^{\prime}}-1\right) \sin ^{2} \phi}{\left(q^{2} \alpha_{H}+q^{\prime 2} / \alpha_{H}-2 q q^{\prime} \cos \phi\right)^{3 / 2}}=\frac{b \tilde{c}_{\mathbf{k}_{H}}}{t \sqrt{x}} \partial_{q} \tilde{p}_{q}$

with $\tilde{c}_{\mathbf{k}_{H}}=c_{\mathbf{k}_{H}}^{\prime}|\sin \phi| \sqrt{6} \cos \Theta_{B} / \sqrt{\pi c_{\mathbf{k}_{H}}}$. For small magnetic fields, $p_{q}$ decays on the scale 1 (both $\alpha_{H}$ and $\phi$ are finite for a generic Fermi surface). This justifies our approximation to expand $G_{\kappa \kappa^{\prime}}$ for large momenta in the regime $\sqrt{t / x}>\max [\sqrt{r} / t, 1]$, i.e. for $t>\max [\sqrt{r x}, x]$. In the original units the width of $p_{\mathbf{k}}$ is approximately
$\Delta k \approx q_{0} t / \sqrt{x}$ and by scaling we obtain the linear resistivity shown in Eqn.(20).

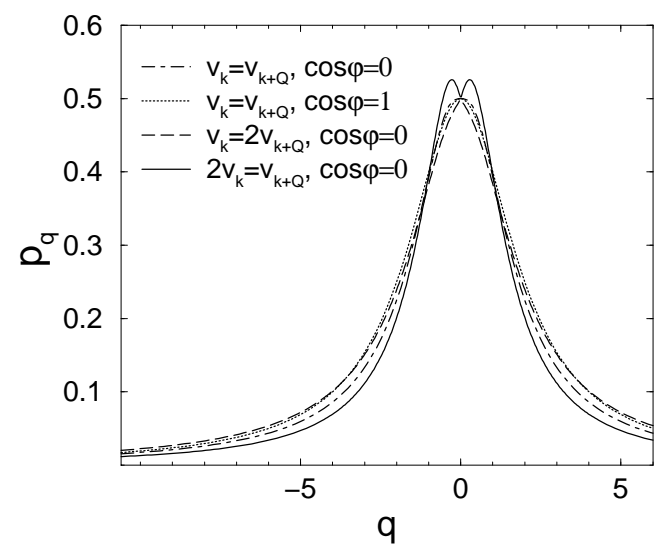

FIG. 8. Shape of $p_{q}$ in the regime $b=0$, $\max [\sqrt{x r}, x]<t<\sqrt{x}$ for various values of $\alpha_{H}=v_{\mathbf{k} \pm \mathbf{Q}} / v_{\mathbf{k}}$ and $\cos \phi=\mathbf{v}_{\mathbf{k} \pm \mathbf{Q}} \cdot \mathbf{v}_{\mathbf{k}} /\left(v_{\mathbf{k} \pm \mathbf{Q}} v_{\mathbf{k}}\right)$. The integral $S=\int p_{q} \approx \pi$ depends very weakly on the parameters. $p_{q}$ with $k \propto q t / \sqrt{x}$ describes how strong spin fluctuations equilibrate the distribution function $\Phi_{\mathbf{k}}$ (9) and $\Phi_{\mathbf{k} \pm \mathbf{Q}}$ in a region of width $t / \sqrt{x}$ around a hot line.

While the kernel of (29) is highly singular, the solution of the integral equation for $b=0$ is nevertheless very smooth: for large momenta $p_{q}$ decays proportional to $1 / q^{2}$ and is finite and smooth for $q \rightarrow 0$ with $p_{0}+\tilde{p}_{0}=1$ to cancel the divergence of the kernel (see figure 8). The perturbation theory in $b /(\sqrt{x} t))$ is analytic and well defined (all eigenvalues of the matrix $\delta\left(q-q^{\prime}\right)\left(1+\int G_{q q^{\prime}} d q^{\prime}\right)+G_{q q^{\prime}}$ are larger than 1) and we obtain for $b<\sqrt{x} t, \sqrt{x}>t>\max [\sqrt{r x}, x]$ :

$$
\frac{\Delta \rho}{\rho_{M}} \sim \frac{b^{2}}{\sqrt{x} t}
$$

Although it is difficult to calculate the precise prefactor analytically, its structure is similar to the prefactor in (25). In Fig. 月 the numerical solution of the Boltzmann equation shows the crossover from the dirty limit (28) to the clean limit (30). In the ultra-clean limit, $x \ll t^{2}$ the distribution function varies on the scale $k_{F}$ and Kohler's rule is valid in leading order and therefore we expect $\Delta \rho \sim b^{2} / t^{2}$.

\section{Magnetoresistivity - large fields}

The diverging prefactors of the $B^{2}$-corrections already indicate that any finite magnetic field will strongly influence the resistivity when the hot lines get sharper and sharper while $B$ drives the quasi-particles over the Fermi surface.

For large fields, the main effect of the $b \partial_{\mathbf{k}} p_{k}$-term in the Boltzmann equation (13) is to keep $\partial_{\mathbf{k}} p_{k}$ small. Due to the boundary conditions $p_{k \rightarrow \pm \infty} \rightarrow 0$ and due to the 
fact that the kernel $G_{k k^{\prime}}$ decays for large momenta, the amplitude of $p_{k}$ decreases for large magnetic fields and $p_{k}$ is broadened. This corresponds to the fact that magnetic field drives the quasi particles over the Fermi surface hence smoothing the quasiparticle distribution. In a strong enough magnetic field we can therefore use perturbation theory in $p_{k}$ and only the first term on the right-hand side of Eqn. (23) survives. In the scaling regime, the resistivity saturates in a large (orbital) magnetic field at a value which is precisely given by the dirtylimit formula (18). In this respect, a magnetic field and disorder have very similar effects, both smooth out the quasi-particle distribution.

As the amplitude of $p_{k}$ decreases for large $b$, the main effect of a large magnetic field in the dirty limit $t<\max [x, \sqrt{r x}]$ is to suppress sub-leading corrections to the conductivity. The maximal change of $\int p_{\kappa}$ is calculated from (23)

$$
\begin{aligned}
\Delta \int p_{\kappa}(b \rightarrow \infty) & \approx \frac{t}{x \sqrt{\alpha_{H}} c_{\mathbf{k}_{H}}} \iint_{-\infty}^{\infty} d \kappa d \kappa^{\prime} G_{\kappa \kappa^{\prime}}\left(p_{\kappa}^{0}+\tilde{p}_{\kappa^{\prime}}^{0}\right) \\
& =\left(1+\alpha_{H}\right) \int_{-\infty}^{\infty} d \kappa\left(p_{\kappa}^{0}\right)^{2}
\end{aligned}
$$

where $p_{\kappa}^{0}=t /\left(x \sqrt{\alpha_{H}} c_{\mathbf{k}_{H}}\right) \int G_{\kappa \kappa^{\prime}} d \kappa^{\prime}$ is the distribution function in the dirty limit in leading order in $t / x$.

The effect of an intermediate field can be calculated by solving the Boltzmann equation in a finite field in perturbation theory in $t / x$ by neglecting again the $G_{\kappa \kappa^{\prime}}\left(p_{\kappa}+\right.$ $\left.\tilde{p}_{\kappa^{\prime}}\right)$ terms.

$$
p_{\kappa} \approx \int_{\kappa}^{\infty} d \kappa^{\prime} p_{\kappa^{\prime}}^{0} \frac{e^{-\left(k^{\prime}-k\right) / \kappa_{b}}}{\kappa_{b}}
$$

with $\kappa_{b}=b /(x \sqrt{t})\left(c_{\mathbf{k}_{H}}^{\prime} /\left(\sqrt{\alpha_{H}} c_{\mathbf{k}_{H}}\right)\right)$. The main effect of the magnetic field is to smear the distribution function on the scale $\kappa_{b}$.

In the Fermi liquid regime $t<\min [r, \sqrt{r x}]$ the scale of the distribution function $p_{\kappa}^{0} \sim(t / x) /\left(r / t+\kappa^{2}\right)$ is given by $\sqrt{r / t}$, accordingly the small-field formula (25) can be applied for $\kappa_{b}<\sqrt{r / t}$ or $b<\sqrt{r} x$. As discussed above, in a large magnetic field $\Delta \rho$ defined in (26) saturates at

$$
\frac{\Delta \rho(b>\sqrt{r} x, t)}{\rho_{M}} \sim \frac{t^{4}}{x r^{3 / 2}} .
$$

and the weak-field expansion (25) connects smoothly to this regime. As above, we can estimate the range of validity of (33) by comparing it to neglected corrections of the order of $\Delta \rho \sim b^{2} \Delta \rho(0, t) / \rho_{0}^{2} \sim b^{2} t^{2} /\left(\sqrt{r} x^{2}\right)$. Therefore (33) is valid for $b<t \sqrt{x / r}, t<\min [x, \sqrt{r x}]$.

The dirty quantum-critical regime $r<t<x$ has a richer structure, as there are two scales in the distribution function $p_{\kappa}^{0}$ (27): 1 and $\max \left[\sqrt{r / t}, e^{-x /(2 t)}\right]$. For $1>\kappa_{b}>\max \left[\sqrt{r / t}, e^{-x /(4 t)}\right]$ the magnetic field, or more precisely $\kappa_{b}^{2}$, takes the role of $r / t$ in (28) and provides a cutoff to the logarithmic divergence of $p_{\kappa}^{0}(27)$. We therefore expect for $r<t<x, \max \left[x \sqrt{t} e^{-x /(2 t)}, \sqrt{r} x\right]<b<$ $x \sqrt{t}$

$$
\frac{\Delta \rho(b, t)}{\rho_{M}} \sim b \frac{t^{2}}{x^{2}}
$$

and the magnetoresistivity will saturate for $b>x \sqrt{t}$ at

$$
\frac{\Delta \rho(b, t)}{\rho_{M}} \sim \frac{t^{5 / 2}}{x}
$$

This has to be compared to the largest sub-leading correction of the form $b^{2} t^{1.5} / x^{2}$ (estimated from Kohler's rule). Therefore equation (35) is valid for $b<\sqrt{t x}$.

All the above discussed effects in the dirty limit are very small on an absolute scale because the disorder has already smeared out most of the features of the distribution function and can only be observed because it is easy to measure the difference $\rho(T, B)-\rho(T)$ with high precision. Much stronger effects are to be expected in the cleaner regime $\max [x, \sqrt{r x}]<t<\sqrt{x}$, where the zero-field resistivity is linear in temperature.

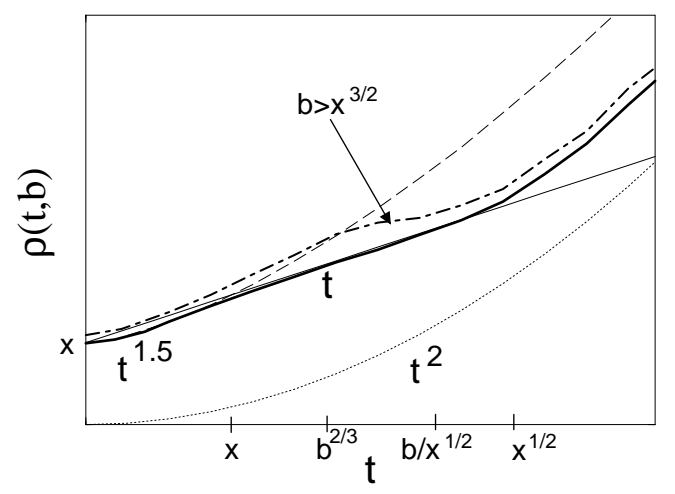

FIG. 9. Schematic plot of the temperature dependence of the resistivity. In an ultra-clean system, the resistivity would rise proportional to $t^{2}$ (thin dotted line). In a system with a small amount $x$ of disorder the resistivity at the QCP (thick solid line) at zero magnetic field rises with $t^{3 / 2}$ at very low temperatures $t<x$, but shows a linear behavior $\Delta \rho \sim \sqrt{x} t$ for $x<t<\sqrt{x}$. If the system is tuned to the quantum-critical point in a finite field, a behavior similar to the dot-dashed line is expected: $\Delta \rho \propto t^{3 / 2}$ should be observed over a larger range $t<b^{2 / 3}$. In an intermediate regime $b^{2 / 3}<t<b / \sqrt{x}$ the resistivity depends weakly on temperature (the thin dashed line corresponds to $\Delta \rho \propto t^{3 / 2}$ ). Note that a different behavior is expected if a finite magnetic field is applied to a compound which is quantum critical for $b=0$ as $b$ drives the system away from the QCP.

For $b<\sqrt{x}$, the effect of the magnetic field can be treated in perturbation theory and (30) is valid. To analyze the regime $\sqrt{x} t<b$ it is useful to rescale the momenta in (29) using $q=-b \tilde{c}_{\mathbf{k}_{H}} /(t \sqrt{x}) k \sqrt{\alpha_{H}}$ so that the Boltzmann equation reads

$$
\sqrt{\alpha_{H}} p_{k}+\frac{t^{2} x}{b^{2} \tilde{c}_{\mathbf{k}_{H}}^{2}} \int \frac{d k^{\prime}\left(p_{k}+\tilde{p}_{k^{\prime}}-1\right) \sin ^{2} \phi}{\left(k^{2}+k^{\prime 2}-2 k k^{\prime} \cos \phi\right)^{3 / 2}}=-\partial_{k} p_{k}
$$


The corresponding equation for $\tilde{p}_{k^{\prime}}$ is obtained by replacing $\alpha_{H}$ by $1 / \alpha_{H}$. The kernel of the integral equation is strongly divergent $\int G_{k k^{\prime}} d k^{\prime} \propto 1 / k^{2}$. The contribution from the integral is, however, small for large magnetic fields and sufficiently large momenta $k>k_{0}$ (a precise estimate is given below) and (36) is solved by $p_{k \gtrless 0}=c_{ \pm} e^{-k \sqrt{\alpha_{H}}}$. For $k<0$ the prefactor $c_{-}$has to vanish due to the boundary condition $p_{|k| \rightarrow \infty}=0$. For $k \rightarrow 0$ the divergence of the kernel dominates the integral equation and forces the solution to take the form $p_{k \rightarrow 0}+\tilde{p}_{k^{\prime} \rightarrow 0}=1$. For $\alpha_{H}=1$ one therefore expects 32 $p_{k \rightarrow 0}=\tilde{p}_{k^{\prime} \rightarrow 0}=1 / 2$. Generally, $p_{k \rightarrow 0}$ is a number close to 0.5 depending slightly on $\alpha_{H}, \phi$ and $b$. For a large magnetic field, the rise of $p_{k}$ close to $k=0$ is independent of $\alpha_{H}$ and $p_{k \rightarrow 0}(b \gg \sqrt{x} t)=\tilde{p}_{k \rightarrow 0}(b \gg \sqrt{x} t)=1 / 2$. The prefactor of the exponential decay $c_{+}$for $k>k_{0}$ is therefore $1 / 2$ and $\int p_{k} d k=1 /\left(2 \sqrt{\alpha_{H}}\right)$ or, after rescaling, $\int p_{\kappa} d \kappa=b /(x \sqrt{t})\left|\cos \Theta_{B}\right|\left(c_{\mathbf{k}_{H}}^{\prime} /\left(2 \alpha_{H} c_{\mathbf{k}_{H}}\right)\right)$. In this regime $\sqrt{x} t<b<\min \left[t^{3 / 2}, t^{2} / \sqrt{r}\right], \max [x, \sqrt{r x}]<t<$ $\sqrt{x}$, the rise of the resistivity $\rho-\rho_{0}$ is linear in $|\mathbf{B}|$ and independent of temperature and disorder (the precise range of validity is calculated below).

$$
\begin{aligned}
\frac{\rho(b, t)-\rho_{0}(b)}{\rho_{M}} \approx & b\left[\frac{3 \pi q_{0}^{3} / 2}{v_{F}^{4}\left((2 \pi)^{3} N_{F}\right)^{2}} \sum_{i} \oint_{i} d \mathbf{k}_{\|}[\right. \\
& \left.\left.\frac{\left(\left(\mathbf{v}_{\mathbf{k}_{\|}}-\mathbf{v}_{\mathbf{k}_{\|} \pm \mathbf{Q}_{i}}\right) \mathbf{n}\right)^{2}\left|\left(\mathbf{v}_{\mathbf{k}_{\|}} \times \mathbf{v}_{\left.\mathbf{k}_{\|} \pm \mathbf{Q}_{i}\right)}\right) \hat{\mathbf{B}}\right|}{\left|\mathbf{v}_{\mathbf{k}_{\|}} \times \mathbf{v}_{\mathbf{k}_{\|} \pm \mathbf{Q}_{i}}\right|}\right]\right]
\end{aligned}
$$

For higher magnetic fields the formula given above will break down, because (29) is valid only for large momenta $\kappa>\max [1, \sqrt{r / t}]$ or, equivalently, $(36)$ is valid for $k>$ $\max [x \sqrt{t} / b, \sqrt{r} x / b]$. To estimate the range of validity of (37), we solve (36) more precisely for $k<0$ in a regime, where $p_{k}$ is still small:

$$
p_{k} \approx \frac{t^{2} x}{b^{2} \tilde{c}_{\mathbf{k}_{H}}^{2}} \int_{-\infty}^{k} d k^{\prime} e^{\left(k^{\prime}-k\right) \sqrt{\alpha}} \frac{2}{k^{2}} \approx \frac{t^{2} x}{b^{2} \tilde{c}_{\mathbf{k}_{H}}^{2}} \frac{2}{k}
$$

$p_{k}$ is large for $k \lesssim k_{0} \approx t^{2} x / b^{2}$ and Eqn. (37) is therefore valid for $k_{0}>\max [\sqrt{t} x / b, \sqrt{r} x / b]$ or $b<$ $\min \left[t^{3 / 2}, t^{2} / \sqrt{r}\right]$. For larger values of $b$ the kernel is less singular and $p_{k}$ stays small for all momenta. Accordingly, the resistivity is dominated by the first term on the righthand side of Eqn. (23) and the magnetoresistivity saturates at a value, which is given by the dirty-limit formula (18):

$$
\begin{aligned}
\frac{\rho(b, t)-\rho_{0}(b)}{\rho_{M}} \approx & t^{3 / 2} h\left(\frac{t}{r}\right) \frac{3 q_{0}^{3}}{8 \pi^{4}\left(v_{F} N_{F}\right)^{2}} \\
& \times \sum_{i} \oint_{i} d \mathbf{k}_{\|} \frac{\left(\left(\mathbf{v}_{\mathbf{k}_{\|}}-\mathbf{v}_{\mathbf{k}_{\|} \pm \mathbf{Q}_{i}}\right) \mathbf{n}\right)^{2}}{\left|\mathbf{v}_{\mathbf{k}_{\|}} \times \mathbf{v}_{\mathbf{k}_{\|} \pm \mathbf{Q}_{i}}\right|} \\
h\left(\frac{t}{r}\right) \approx & \begin{cases}\frac{\pi \zeta(3 / 2) \Gamma(5 / 2)}{2 \sqrt{2}}, t>r \\
\frac{\pi^{3}}{12} \sqrt{t / r} & , t<r\end{cases}
\end{aligned}
$$

This expression is valid for $\min \left[t^{3 / 2}, t^{2} / \sqrt{r}\right]<b<$ $\min \left[t^{1 / 4} x^{3 / 4}, \sqrt{t} x^{3 / 4} / r^{1 / 4}\right]$, where the upper limit estimates the regime where non-universal contributions following Kohler's rule dominate. In Fig. 9 the temperature dependence of the large-field magnetoresistivity is shown schematically.

\section{DISCUSSION}

\section{A. Overview over magnetoresistivity}

Before we discuss the experimental relevance of our calculations in detail, we collect the results for the magnetoresistivity in the scaling limit (12). Most of the prefactors, which are omitted here, can be found in the previous sections. Only orbital effects of the magnetic field are taken into account, a discussion of the interplay of spin and orbital effects is given in section IV $t$ and $b$ defined in (5) are the dimensionless temperature and magnetic field, respectively. $x$ is proportional to the strength of disorder and can crudely be identified with the inverse of the residual resistivity ratio and $r$ measures the distance from the QCP.

In the disorder dominated regime the absolute size of effects of an orbital magnetic field are quite small. Close to the QCP, for $r \ll t<x$, the prefactor of the $b^{2}$-term is, however, extremely large and for very small magnetic fields a crossover to a linear field dependence of the magnetoresistivity is predicted. For $\Delta \rho=\rho(b, t)-\rho(b, 0)$ we obtain

$$
\frac{\Delta \rho}{\rho_{M}} \sim t^{3 / 2}-\frac{t^{5 / 2}}{x}+ \begin{cases}\frac{b^{2} t^{2}}{x^{2} g_{x, r, t}} & , b<g_{x, r, t} \\ b \frac{t^{2}}{x^{2}} & , g_{x, r, t}<b<x \sqrt{t} \\ \frac{t^{5 / 2}}{x} & , x \sqrt{t}<b<\sqrt{t x}\end{cases}
$$

with $g_{x, r, t} \approx \max \left[x \sqrt{r}, x \sqrt{t} e^{-x /(4 t)}\right]$. For very small $g_{x, r, t}$ this expression can be used to measure the temperature dependence of the correlation length $\xi$ using $r \propto 1 / \xi^{2}$.

In the disorder-dominated Fermi liquid regime $t<$ $\min [r, \sqrt{r x}]<1$, the temperature dependent part of the magnetoresistivity is still enhanced, but it is probably very difficult to extract the $t^{4}$ contribution experimentally.

$$
\frac{\Delta \rho}{\rho_{M}} \sim \frac{t^{2}}{\sqrt{r}}-\frac{t^{4}}{x r^{3 / 2}}+\left\{\begin{array}{l}
\frac{b^{2} t^{4}}{x^{3} r^{5 / 2}}, b<\sqrt{r} x, t>r \sqrt{x} \\
\frac{t^{4}}{x r^{3 / 2}} \quad, \sqrt{r} x<b<t \sqrt{\frac{x}{r}}
\end{array}\right.
$$

The largest effects can be observed for rather clean systems with $\max [x, \sqrt{r x}]<t<\sqrt{x}$, which show a linear resistivity for $b=0$ : 


$$
\frac{\Delta \rho}{\rho_{M}} \sim \begin{cases}\sqrt{x} t+\frac{b^{2}}{\sqrt{x} t} & , b<\sqrt{x} t \\ b \quad & \sqrt{x} t<b<\min \left[t^{3 / 2}, \frac{t^{2}}{\sqrt{r}}\right] \\ \min \left[t^{3 / 2}, \frac{t^{2}}{\sqrt{r}}\right], & \min \left[t^{3 / 2}, \frac{t^{2}}{\sqrt{r}}\right]<b< \\ & x^{3 / 4} \min \left[t^{1 / 4}, \frac{\sqrt{t}}{r^{1 / 4}}\right]\end{cases}
$$

\section{B. Experimental situation for $B=0$}

According to our calculation, in $d=3$, spin-fluctuation theory predicts that the resistivity in dirty systems rises with $T^{3 / 2}$, while in cleaner compounds a large regime with a linear resistivity is expected. This behavior is actually seen in a large number, but not all, AFM quantumcritical systems. A resistipity proportional to $T^{1.5}$ has been reported in $\mathrm{CeCu}_{2} \mathrm{Si}_{2} \mathrm{U}$, $\mathrm{CeNiGa}_{2} \mathrm{O}, \mathrm{Cefu}_{6-x} \mathrm{Ag}_{x} \mathrm{LO}$ $\mathrm{CeNi}_{2} \mathrm{Ge}_{2}$, $\mathrm{CePd}_{2} \mathrm{Si}_{2}, \mathrm{CeIn}_{3} 5$ or $\mathrm{CeCu}_{5} \mathrm{Au} 11$. All these systems are dirty in the following sense: in the regime, where the exponent 1.5 has been observed, the rise of the resistivity $\Delta \rho$ is small compared to the residual resistivity.
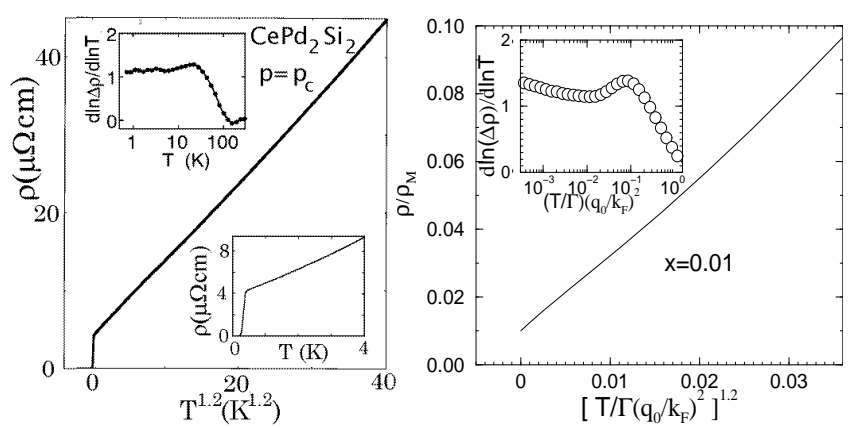

FIfr. 10. Resistivity as a function of $T^{1.2}$ in $\mathrm{CePd}_{2} \mathrm{Si}_{2}$ taken from (left figure) compared to our calculation within model A defined in appendix $D$ for $x=0.01$ (right figure). The insets show the corresponding logarithmic derivative of $\rho(T)-\rho(0)$. If one compares the insets one has to take into account that the low-temperature behavior of the logarithmic derivative of $\Delta \rho$ strongly depends on the exact determination of $\rho_{0}$ (see inset of Fig. (4). Below $\approx 400 \mathrm{mK} \mathrm{CePd}_{2} \mathrm{Si}_{2}$ is superconducting (lower inset). Note the offset of the line $T=0$ in both plots.

For a small number of the above mentioned compounds, cleaner samples exist. For these high-purity single crystals, the resistivity seems to rise with an exponent smaller than 1.5. The cleaner the samples are, the closer the exponent is to 1 . This qualitative trend coincides with the predictions of our calculations(Fig. 3). Examples for this are $\mathrm{CePd}_{2} \mathrm{Si}_{2}, \mathrm{CeNi}_{2} \mathrm{Ge}_{2}$. 5 . 3 where samples with RRR of the order of 100 exist. In clean samples of $\mathrm{CeIn}_{3}$, exponents smaller than 1.5 are observed over some temperature range, but at lowest temperatures the exponent crosses over to 1.5 , as expected from theory. In Fio. 10 the resistivity of a quite clean sample of $\mathrm{CePd}_{2} \mathrm{Si}_{2} \mathrm{I}$ is compared to a typical solution of the Boltzmann equation. It is important to point out, that not only a similar effective exponent shows up in both theory and experiment, but that it is observed over a similar range $0.1 \rho_{0}<\Delta \rho(T)<10 \rho_{0}$. Only the effective scale $\Gamma / q_{0}^{2}$ seems to be approximately a factor 10 too large compared to $T_{K} / k_{F}^{2}$, but a quantitative comparison of these high-energy scales is certainly very dubious within our perturbative treatment of a strongly correlated system. Furthermore, a quantitative comparison would require a more realistic model based on some knowledge of the band structure and the actual width of the spinfluctuation.

The theory, presented in this paper, is certainly not applicable to strongly disordered systems, where the nature of the quantum-critical transition is changed due to disorder. It does not explain the linear temperature dependence of the resistivity in $\mathrm{CeCu}_{6-x} \mathrm{Au}_{x}$ due to two reasons. The first is, that $\mathrm{CeCu}_{6-x} \mathrm{Au}_{x}$ is relatively dirty at the QCP $(x=0.1)$. More important is the unusual nature of the spin-fluctuations, which are strongly anisptropic 38 , and show an unusual frequency dependence 18 .

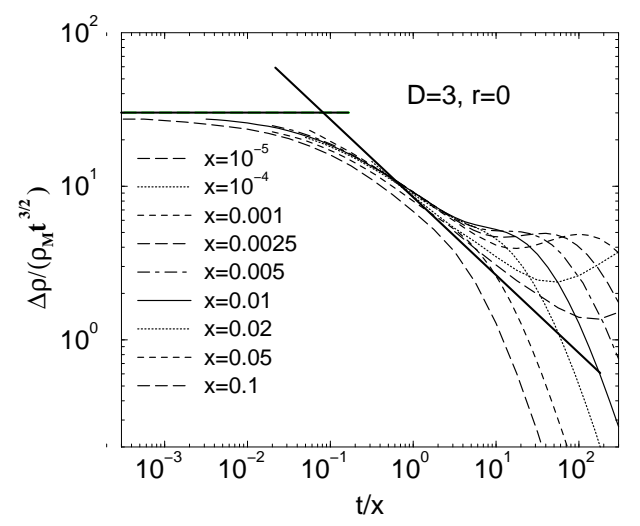

FIG. 11. Scaling plot of $f(t / x, 0)=\Delta \rho(t / x) / t^{3 / 2}$ at the QCP $(r=0)$ using a numerical solution of the Boltzmann equations for various amounts of disorder $x$. The straight solid lines show the limiting cases $\Delta \rho \propto t^{3 / 2}$ for $t \ll x$ and $\Delta \rho \propto t$ for $x \ll t \ll \sqrt{x}$. Deviations from scaling are large for $t / x>1 / \sqrt{x}$.

The most precise test of the spin-fluctuation model would be to verify (or falsify) our scaling prediction (11) by comparing several very clean quantum-critical samples with varying residual resistivity ratios. An example for the expected deviations from scaling is shown in Fig. 11.

A somewhat unexpected result of our calculation is that in clean systems away from the QCP, a Fermi liquid form of the resistivity can be observed only at very low temperatures $t<\sqrt{r x}$. This has to be compared to thermodynamic quantities, where FL behavior sets in at a considerably higher scale $t<r$. In a clean system with 
a RRR over 100, the crossover scale in transport quantities can easily be e.g. a factor 5 lower than in thermodynamic quantities. This might explain claims based on transport measurements that the non-Fermi liquid behavior in these cleansystems extends over a finite region away from the QCPG,

\section{Spin- and orbital effects of $B$}

A magnetic field influences the resistivity near a quantum critical point in two quite independent ways: by spin- and orbital effects. The spin contribution typically suppresses the antiferromagnetic order. In the context of this paper, we will call these phenomena "spin effects" despite the fact, that in most cases orbital degrees of freedom are involved. On the non-magnetic side of the phase diagram and for $B$ fields, which are not too large, the leading order spin-effects can supposedly be described by the magnetic field dependence of $r$, the variable which describes the distance to the QCP. For small fields, one expects in an AFM metal above the upper critical dimension $\Delta r \propto B^{2}$. A controlled study of the effects of a finite magnetic field close to the AFM QCP in a metal does not exist to our knowledge, however, Ioffe and Millis 39 have carefully investigated the uniform susceptibility without finding unexpected anomalies. A large magnetic field can induce other (first-order) transitions or change the nature of the magnetic fluctuations completely.

The focus of this paper are "orbital effects". We consider a regime where interference effects don't play any role and have restricted our attention to the classical $\mathbf{v} \times \mathbf{B}$ force on the electrons. This approximation is valid in the scaling limit $(12)$, where $\omega_{c} \tau \ll 1$ and the disorder is so weak that weak-fogalization corrections set in at much lower temperatures 36 .

In the scaling limit (12), i.e. for clean enough systems at very low temperatures in small magnetic fields close to the QCP, spin effects can be neglected. For $b \propto x^{3 / 2}$ the correction $\Delta r \propto b^{2}$ is small compared to $x$ for sufficiently small $x$. In realistic heavy Fermion systems, it is, however, probably not possible to produce a sample which is clean enough so that only orbital effects show up in the interesting regime. The reason is that on the one hand the Lorentz force $\mathbf{v} \times \mathbf{B}$ is suppressed due to the large mass $m^{*}$ of the heavy quasiparticles and on the other hand spin-effects are enhanced due to the large susceptibility $\chi \approx 1 / T_{K} \propto m^{*} / m_{0}$.

A reliable estimate of these effects is difficult as the details of the interplay of the Kondo effect and the RKKY interaction are poorly understood 40 . For a crude estimate, we assume that all high-energy scales, including scattering rates and renormalized coupling constants can be approximated by $T_{K}$. Orbital effects are important for $b>x^{3 / 2}$ or $\mu_{B} B>x^{3 / 2} k_{B} T_{K} m^{*} / m_{0}$ and spin effects can be neglected for $\Delta r<x$ or $\mu_{B} B<\sqrt{x} k_{B} T_{K} \sqrt{m_{0} / m^{*}}$. If this is qualitatively correct, only an extremely clean sample with $x<\left(m_{0} / m^{*}\right)^{3 / 2}$, i.e. with a RRR larger than $\left(m^{*} / m_{0}\right)^{3 / 2}$, shows orbital effects without the influence of spin effects. This implies that spin-effects are present in most cases, because $m^{*} / m_{0}$ is quite large in those clean stoichiometric systems where the quantum critical point is reached by pressure.

Nevertheless, the orbital effects have a very clear signature even in the presence of large spin-effects, especially in suitably designed experiments. The necessary magnetic fields are not very large. In our units, Shubnikov-de Haas oscillations are expected for $b>x$ at low temperatures while strong effects in the magnetoresistivity in clean samples set in for $b>x^{3 / 2}$, i.e. for magnetic fields which can easily be an order of magnitude smaller.

The Hall constant is obviously not influenced by $\mathrm{O}\left(B^{2}\right)$ corrections due to the suppression of the AFM and so $R_{H}$ is not affected by the above mentioned problems. Therefore it should be very interesting to investigate the strong sample dependence of the Hall effect predicted in section III A. Unfortunately, only extremely clean samples will show a strong temperature dependence (see Fig. 6) 41.

For a detailed study of the magnetoresistivity in clean systems, it is necessary to minimize spin effects. In a system like $\mathrm{CePd}_{2} \mathrm{Si}_{2}$ the effective distance from the QCP can be tuned by pressure. Therefore, one can select various pressures on the antiferromagnetic side of the phase diagram close to the QCP and suppress the remaining magnetic order by a suitable magnetic field. For such a set-up the spin effects are compensated by a change of pressure to a large extent especially in systems with Ising symmetry. For not too strong fields the field-tuned quantum-critical transition should stay second order and one can compare several quantum-critical systems $(r=0)$ with the same amount of disorder $x$ but different magnetic fields. Our calculation predicts, that in a magnetic field $b>x^{3 / 2}$, the low-temperature resistivity rises proportional to $t^{1.5}$. A schematic plot of the expected behavior is shown in Fig. 9. Ideally, one can test the scaling predictions by comparing samples of different quality. In an anisotropic system one should choose a direction of the magnetic field perpendicular to the easy axis and to one of the ordering vectors $\mathbf{Q}$, because only the component of $B$ parallel to the hot lines enters the scaling form of the resistivity. (This effect is described

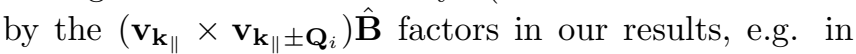
(37).)

A simple way to distinguish spin- and orbit effect is the sign of the magnetoresistivity. A suppression of the AFM fluctuations on the paramagnetic side of the phase diagram will reduce the amount of scattering and the resistivity will go down. Due to the orbital effects discussed in the previous section resistivity increases because $b$ smears out the quasi particle distribution, therefore minimizing the effect that cold regions short-circuit the hot spots. This can lead to a sign change of $\Delta \rho=$ $(\rho(B, T)-\rho(0, T))-(\rho(B, 0)-\rho(0,0))$ either as a function of disorder or strength of the magnetic field. Such an 
observation could help to separate the two effects quantitatively.

The interplay of the spin- and orbit effects can also lead to less trivial effects. In the dirty limit close to the QCP e.g. the magnetoresistivity for small magnetic fields (28) is proportional to $1 / \sqrt{r}, \Delta \rho \sim b^{2} t^{2} /\left(x^{3} \sqrt{r}\right)$. If spin effects suppress the AFM fluctuations with $r \approx c b^{2}$, one can expect a regime where the magnetoresistivity rises linearly with $B$ for rather small fields $\left(c b^{2}<r(b=\right.$ $0, t)), \Delta \rho \propto b t^{2} /\left(\sqrt{c} x^{3}\right)$. It is probably not very easy to distinguish this effect from the linear $b$ dependence due to orbital effects discussed in (34) with $\Delta \rho \sim b t^{2} / x^{2}$ for $b>x \sqrt{r(b=0)}$.

In high purity single crystals of $\mathrm{CeNi}_{2} \mathrm{Ge}_{2}$ Grosche et al. have reported measurements of the resistivity in magnetic fields of a few Tesla. As explained above, a direct comparison of their findings with our calculations is difficult due to the interplay of orbital and spin effects. However, an important qualitative trend of their results can easily be explained by our approach. In finite magnetic field, they observe a clear $T^{2}$ dependence below a temperature $T^{*}(B)$. Obviously, the magnetic field drives the system away from the quantum-critical point. Remarkably, it seems that the magnetic field is much more effective in this respect than pressure, which also suppresses the magnetic order. For samples tuned by pressure towards the paramagnetic side of the phase diagram, a clear $T^{2}$-rise of the resistivity could not be observed. This qualitative effect can be understood within our approach. In the case of pressure tuning, we expect Fermi liquid in a very clean sample only for very low temperatures $t<\sqrt{r x}$. In a sufficiently strong magnetic field, the crossover to $T^{2}$ should be visible at a much higher temperature $t<r^{1 / 4} \sqrt{b}$ in agreement with the experimental trends. If we assume that $r \propto b^{2}$ and use the above discussed crude estimates of the prefactors, we expect the crossover temperature $T^{*}$ to rise linear in $B$ with $k_{B} T^{*} \approx \mu_{B} B\left(m_{0} / m^{*}\right)^{1 / 4}$. I want to emphasize that this estimate is unreliable and should be replaced by a suitable strong-coupling theory of heavy-Fermion systems. Nevertheless, the experimental results 8 seem to be consistent with this simple-minded estimate of scales.

\section{CONCLUSION}

In all situations where two scattering mechanisms compete, one anisotropic and affecting only parts of the Fermi surface, the other isotropic, the transport is quite subtle and the interplay of the two mechanisms, $A$ and $B$, can lead to qualitatively new effects. The reason for this is very simple: the effectiveness of scattering mechanism $A$ in the presence of a current is strongly influenced by the momentum dependence of the out-of-equilibrium distribution of the quasi particles, which is affected by mechanism $B$. Therefore, we expect that $\rho_{A B} \neq \rho_{A}+\rho_{B}$ even in the weak-coupling limit of a semi-classical theory de- scribed by a Boltzmann equation ( $\rho_{X}$ is the resistivity in the presence of scattering mechanism $X)$.

In this paper we have analyzed such a situation in detail. Near the AFM quantum-critical point of slightly disordered metals, weak isotropic impurity scattering competes with the strongly anisotropic scattering from spin fluctuations, which is most effective along "hot" lines on the Fermi surface. The observation that even in relatively clean single crystals the effective resistivity exponent $\alpha, \Delta \rho \propto T^{\alpha}$, depends on the amount of disorder in the system $5.8,8$, is an important indication that the interplay of scattering mechanisms is relevant in these systems. Our results for $B=0$ explain the experimental trends: $\Delta \rho \propto T^{1.5}$ for dirty systems and $\Delta \rho \propto T$ in high purity samples. A precise test of the spinfluctuation picture which underlies our calculation would be to check our scaling predictions using a comparison of several high-purity samples with different residual resistivities $\rho_{0}$ directly at the QCP: $\Delta \rho \approx T^{1 / 5} f\left(T / \rho_{0}\right)$ with $f(y \rightarrow 0)=$ const. and $f(y \rightarrow \infty) \propto 1 / \sqrt{y}$.

We furthermore propose to use orbital effects in a large magnetic field to study anisotropic scattering mechanisms in detail. Interesting nonlinear effects are expected if the magnetic field is strong enough to push quasi particles within the scattering time $\tau_{\mathbf{k}}$ over any sharp structure with width $\Delta k$ on the Fermi surface, i.e. for $e(\mathbf{v} \times \mathbf{B}) \tau_{\mathbf{k}}>\Delta k$. In the limit of very small but finite disorder, we were able to calculate the non-linear magnetoresistivity analytically in all relevant limits. The main effect of a magnetic field is that sharp features in the quasiparticle distribution are smeared out. In this respect both an increase of disorder and a magnetic fields lead to similar effects. In a sufficiently strong magnetic fields, we therefore expect a $T^{1.5}$ rise of the resistivity at the QCP and a $T^{2}$ law on the Fermi-liquid side of the phase diagram even in samples where the resistivity is close to linear in the absence of the magnetic field. Precise scaling predictions can be used to check this mechanism experimentally.

We hope that the results of this paper establish a set of predictions which can be used to investigate mainly one question: Can the non-Fermi liquid behavior in clean systems be explained by a nearly antiferromagnetic Fermi liquid? At the moment this question is still open. Our interpretation of the data e.g. of $\mathrm{CePd}_{2} \mathrm{Si}_{2}$ favors a SDW interpretation. Certainly it is necessary to look also at other quantities. For example in $\mathrm{CePd}_{2} \mathrm{Si}_{2}$ the Neél temperature seems to rise linearly with $p-p_{c}$ while in spinfluctuation theory an exponent $2 / 3$ is expected.

It is important to stress that at least in some systems close to an AFM QCP a description within the threedimensional SDW scenario seems to be impossible. The best studied example for this is $\mathrm{CeCu}_{5.9} \mathrm{Au}_{0.1}{ }^{3}$ where the spin-fluftuations spectrum shows both an anomalous momentum 38 and energy 18 dependence. The linear temperature dependence of the resistivity in $\mathrm{CeCu}_{5.9} \mathrm{Au}_{0.1}$ has a different origin than the one discussed in this paper. As the residual resistivity is relatively high, the resistiv- 
ity should rise with $T^{1.5}$ within the $3 d$ models discussed in this paper (a linear resistivity could resylt from $2 d$ critical fluctuations coupled to $3 d$ electrons 38 ). Interestingly, an exponent 1.5 has been reperted for the quantum critical resistivity of $\mathrm{CeCu}_{6-x} \mathrm{Ag}_{x} 10$ and pressure tuned $\mathrm{CeCu}_{5} \mathrm{Au} 11$, which might indicate that small changes can induce a more conventional SDW-transition in this compound. $\mathrm{CeCu}_{5.9} \mathrm{Au}_{0.1}$ is by now the most likely and best studied candidate 18.20 . 19 for a trulyunconventional QCP $\left(\mathrm{YbRh}_{2} \mathrm{Si}_{2} 12\right.$ and $\mathrm{U}_{0.2} \mathrm{Y}_{0.8} \mathrm{Pd}_{2} \mathrm{Al}_{3} 13$ have very similar thermodynamic and transport properties). The re ported scaling of the susceptibility in $\mathrm{CeCu}_{5} \mathrm{Au}_{0.1} 18$ seems to contradict any simple SDW models38. An interesting question is whether the rather strong disorder is responsible for the anomalous behavior or whether a pure interaction effect destroys the Fermi liquid close to the QCP.

\section{ACKNOWLEDGEMENT}

I would like to thank N. Andrei, R. Chitra, A.V. Chubukov, P. Coleman, P. Gegenwart, M. Grosche, S. Julian, G. Kotliar, E. Lange, H. v. Löhneysen, A. Millis, C. Pfleiderer, A.E. Ruckenstein and P. Wölfle for discussions. This work was supported by the A. v. Humboldt foundation and the Center for Materials Theory at Rutgers University.

\section{APPENDIX A: APPROXIMATE VARIATIONAL SOLUTION OF THE BOLTZMANN EQUATIONS}

In the absence of the magnetic field the Boltzmann equation is equivalent to the following variational problem 12,27 which allows to calculate the qualitative behavior of the resistivity in a yery simple and efficient way. Following Hlubina and Rice 27, we define a functional $\rho$ of $\Phi_{\mathbf{k}}$

$$
\rho\left[\Phi_{\mathbf{k}}\right]=\frac{\hbar}{4 e^{2}} \frac{\oint \oint \frac{d \mathbf{k} d \mathbf{k}^{\natural}}{v_{\mathbf{k}} v_{\mathbf{k}^{\prime}}} F_{\mathbf{k}^{\prime}}\left(\Phi_{\mathbf{k}}-\Phi_{\mathbf{k}^{\prime}}\right)^{2}}{\left(\oint \frac{d \mathbf{k}}{v_{\mathbf{k}}}\left(\mathbf{v}_{\mathbf{k}} \mathbf{n}\right) \Phi_{\mathbf{k}}\right)^{2}} \longrightarrow \min
$$

The physical resistivity is given by the minimum of $\rho\left[\Phi_{\mathbf{k}}\right]$ regarded as a functional of $\Phi_{\mathbf{k}}$, the scattering matrix $F$ is defined in (8). Instead of solving the Boltzmann equation exactly, we can guess the qualitative structure of the solution: the strong scattering will equilibrate the distribution function in a region of width $\Delta k$ around the hot lines (see Fig. 11). In the dirty limit, the distribution function is of the well known form $\Phi_{\mathbf{k}}^{0}=\mathbf{v}_{\mathbf{K}} \mathbf{E}$ and as a variational ansatz we assume that $\Phi_{\mathbf{k}}$ vanishes (or more precisely is equal to $\left.1 / 2\left(\Phi_{\mathbf{k}}^{0}+\Phi_{\mathbf{k} \pm \mathbf{Q}}^{0}\right)\right)$ in a region of width $\Delta k$ around the hot lines. It is easy to calculate the qualitative structure of $\Delta \rho$ as a function of the variational parameter $\Delta k$ in the regime where the disorder dominates $\Delta \rho \lesssim \rho_{0}$ :
$\Delta \rho[\Delta k] \approx x|\Delta k|+\frac{t^{2}}{\left(t+r+(\Delta k)^{2}\right)^{(4-d) / 2}}+O\left(t^{2}\right)$

The first term is due to the denominator in (A1) and reflects the fact that the disorder favors a smooth distribution function. The structure of the second term can easily be guessed (or calculated). It reproduces the well-known results e.g. of Moriya 17 for $\Delta k=0$, who essentially averages the scattering rates $F_{\mathbf{k k}^{\prime}}$ over the full Fermi surface.

In the dirty limit, the first term in (A2) dominates, $\Delta k$ vanishes at the minimum and one obtains the well-known

$$
\Delta \rho \approx \frac{t^{2}}{(t+r)^{(4-d) / 2}}, \quad \text { for } \Delta k \ll t+r .
$$

In the ultra-clean limit $\left(x \ll t^{2}\right), \Delta k$ is of order $k_{F}$ and

$$
\Delta \rho \approx t^{2} \text { for } \Delta k \approx 1
$$

For clean systems there is an interesting intermediate regime where $t+r<(\Delta k)^{2}<1$, where we obtain a non-trivial result by minimizing ( $\mathrm{A} 2$ )

$$
\Delta \rho \approx t^{\frac{2}{5-d}} x^{\frac{4-d}{5-d}} \quad \text { with } \sqrt{t+r}<\Delta k \approx\left(t^{2} / x\right)^{\frac{1}{5-d}}<1 .
$$

This very simple calculation reproduces the results of (21) qualitatively.

\section{APPENDIX B: RELAXATION TIME APPROXIMATIONS}

Strictly speaking, a relaxation time approximation is not valid near a quantum critical point, where the scattering is dominated by processes involving a fixed momentum transfer $\mathbf{Q}$. The time to equilibrate a quasiparticle distribution at the point $\mathbf{k}$ on the Fermi surface will crucially depend on the quasiparticle distribution at the point $\mathbf{k}+\mathbf{Q}$. For example if $\mathbf{Q}$ is perpendicular to the electric field $\mathbf{E}$ and some reflection or gliding plane (and if the Fermi surface is not too complicated), then $\mathbf{Q}$ will connect points with the same non-equilibrium quasiparticle distribution and the singular scattering will not relax the non-equilibrium distribution. In such a situation vertex corrections cancel the leading self-energy contributions and the relaxiation time approximation is completely wrong (see also [ 37] on the role of vertex corrections for the Hall effects).

Nevertheless, the relaxation time analysis gives qualitatively the correct resistivity for generic situations, where the $\mathbf{Q}$-vectors are not perpendicular to $\mathbf{E}$ as can be verified by comparison with the full solution of the Boltzmann equations discussed in the main text. The relaxation time approximation can have much larger errors for the Hall effect because the distribution function $\Phi_{\mathbf{k}}$ perpendicular to the current is often dominated by quasiparticles which are scattered into this region by spinfluctuations with momentum $\mathbf{Q}_{i}$ (in the geometry show in Fig. 1 this effect is absent). 
In second order perturbation theory the life-time of the electron at $T=0$ is given by 27

$$
\frac{1}{\tau_{\mathbf{k}}}=2 g_{S}^{2} / \Gamma \sum_{\mathbf{k}^{\prime}} \int_{0}^{\epsilon_{\mathbf{k}}} d \omega \operatorname{Im} \chi_{\mathbf{k}-\mathbf{k}^{\prime}}(\omega) \delta\left(\omega-\left(\epsilon_{\mathbf{k}}-\epsilon_{\mathbf{k}^{\prime}}\right)\right),
$$

where $\chi$ is given by (2) with $\omega_{\mathbf{q}}=\left(\mathbf{q} \pm \mathbf{Q}_{i}\right)^{2} / q_{0}^{2}$. Splitting the integration over $\mathbf{k}^{\prime}$ in a surface integral over the Fermi surface and an energy integral perpendicular to it $\int d^{3} \mathbf{k}^{\prime}=\iint d \mathbf{k}^{6} / v_{F} \int d \epsilon_{\mathbf{k}^{\prime}}$, where $v_{F}\left(\mathbf{k}^{\prime}\right)$ is the Fermi velocity, performing first the $\epsilon_{\mathbf{k}^{\prime}}$ and then the $\omega$ integration we obtain

$$
\begin{aligned}
\frac{1}{\tau_{\mathbf{k}}} & \approx \frac{g_{S}^{2}}{v_{F}(2 \pi)^{3}} \iint d \mathbf{k}^{\prime} \ln \left[\frac{\left(\epsilon_{\mathbf{k}} / \Gamma\right)^{2}+\left(r+\omega_{\mathbf{k}-\mathbf{k}^{\prime}}\right)^{2}}{\left(r+\omega_{\mathbf{k}-\mathbf{k}^{\prime}}\right)^{2}}\right] \\
& \approx \frac{g_{S}^{2} q_{0}^{2}}{v_{F}(2 \pi)^{2}} \frac{\epsilon_{\mathbf{k}}}{\Gamma} \min \left[\frac{\epsilon_{\mathbf{k}}}{2 \Gamma \delta_{k}^{2}}, 1\right], \quad \delta_{k}^{2}=r+\left(\delta \mathbf{k} / q_{0}\right)^{2}
\end{aligned}
$$

where $\delta \mathbf{k}$ is the distance of $\mathbf{k}+\mathbf{Q}$ from the Fermi surface, i.e. essentially the distance of $\mathbf{k}$ from lines on the Fermi surface, where $\epsilon_{\mathbf{k}}=\epsilon_{\mathbf{k} \pm \mathbf{Q}_{i}}=\mu$. Along these "hot lines" the inelastic scattering is strongest.

The scattering rate depends crucially on the distance from the hot lines $\delta \kappa=\delta k / k_{F} \approx \delta k / q_{0}$. Far from the hot lines or at lowest energies for a system not directly at the quantum critical point, we recover the usual $1 / \tau_{\mathbf{k}} \propto \epsilon_{\mathbf{k}}^{2}$. Directly at the QCP and for $\delta \kappa=0$ the scattering rate is linear in the quasiparticle energy. At finite temperatures a typical quasi particle has the energy $T$ and for qualitative estimates we use the approximation

$$
\frac{1}{\tau_{\mathbf{k}}^{s}} \approx \frac{1}{\tau_{M}} \begin{cases}t^{2} /(r+t) & \text { for } \delta \kappa<\sqrt{r+t} \\ t^{2} /(\delta \kappa)^{2} & \text { for } \delta \kappa>\sqrt{r+t}\end{cases}
$$

where $\tau_{M}$ is a typical scattering rate at the temperature $\Gamma$ and $t=T / \Gamma$ the dimensionless temperature. For $r \ll 1$ the scattering is highly anisotropic and strongest in a region of width $\delta \kappa \approx \sqrt{r+t}$. In the presence of weak disorder, the elastic scattering due to impurities has to be added

$$
\frac{1}{\tau_{\mathbf{k}}}=\frac{1}{\tau_{\mathrm{el}}}+\frac{1}{\tau_{\mathbf{k}}^{s}} \approx x \frac{1}{\tau_{M}}+\frac{1}{\tau_{\mathbf{k}}^{s}}
$$

where me measure the relative strength of impurity scattering by the dimensionless quantity $x \approx \tau_{M} / \tau_{\mathrm{el}} \approx$ $1 / \mathrm{RRR}$ which is defined in (5).

The conductivity in relaxation time approximation is proportional to the average of $\tau_{\mathbf{k}}$ over the Fermi surface (assuming that the Fermi velocity does not vary too strongly). By integrating ( $(\mathrm{B} 4)$ over $\delta \kappa$, we therefore obtain an estimate for the conductivity. The conductivity $\sigma$ in units of the conductivity $\sigma_{M}=1 / \rho_{M}$ is approximately given by

$$
\begin{aligned}
\frac{\sigma(T)}{\sigma_{M}} & \approx\left\langle\tau_{\mathbf{k}}\right\rangle / \tau_{M}=\left\langle\left(\frac{\tau_{M}}{\tau_{\mathrm{el}}}+\frac{\tau_{M}}{\tau_{\mathbf{k}}^{s}}\right)^{-1}\right\rangle \\
& \approx \int_{0}^{\sqrt{r+t}} d \delta \kappa \frac{1}{x+t^{2} /(r+t)}+\int_{\sqrt{r+t}}^{1} d \delta \kappa \frac{1}{x+t^{2} /(\delta \kappa)^{2}}
\end{aligned}
$$

$$
\begin{aligned}
=\frac{1}{x}( & -\frac{t^{2} / \sqrt{r+t}}{x+t^{2} /(r+t)} \\
& \left.-\frac{t}{\sqrt{x}}\left(\arctan \frac{\sqrt{x}}{t}-\arctan \frac{\sqrt{x} \sqrt{r+t}}{t}\right)\right)
\end{aligned}
$$

In the ultra-clean limit, $x \ll t^{2}<1$, the main contribution arises from regions far from the hot line or large $\delta \kappa$. The conductivity diverges with $1 / T^{2}$ with a prefactor of order 1

$$
\rho(\Gamma t) \approx t^{2} \text { for } \frac{1}{\tau_{\mathrm{el}}} \ll \min \left[\frac{1}{\tau_{\mathbf{k}}^{s}}\right] \text { or } x \ll t^{2} .
$$

as has been pointed out by Hlubina and Rice27. In the dirty limit, $x \gg t^{2} /(r+t)$, the elastic scattering dominates and one can expand (B6) or (B7) in the inelastic scattering rates:

$$
\begin{aligned}
\frac{\Delta \rho(T)}{\rho_{M}} \approx \tau_{M}\left\langle\frac{1}{\tau_{\mathbf{k}}^{s}}\right\rangle & \approx 2 \frac{t^{2}}{\sqrt{r+t}} \\
\text { for } \frac{1}{\tau_{\mathrm{el}}} & \gg \max \left[\frac{1}{\tau_{\mathbf{k}}^{s}}\right] \text { or } t \ll \max [\sqrt{r x}, x]
\end{aligned}
$$

and we recover the well known $\delta \rho \propto T^{3 / 2}$ near the quantum-critical point.

In the relaxation time approximation we obtain similar to our results for the Boltzmann equation a pronounced intermediate regime, where $\min \left[1 / \tau_{\mathbf{k}}^{s}\right] \lesssim 1 / \tau_{\text {el }} \lesssim$ $\max \left[1 / \tau_{\mathbf{k}}^{s}\right]$. This corresponds to the regime where the first arctan in (B7) is $\pi / 2$, while the second is small, i.e. $\max [\sqrt{r x}, x]<t<\sqrt{x}$. The rise of the resistivity in linear in temperature in this regime:

$$
\frac{\Delta \rho(T)}{\rho_{M}} \approx t \frac{\pi}{2} \sqrt{x} \text { for } \max [\sqrt{r x}, x]<t<\sqrt{x} .
$$

\section{APPENDIX C: NUMERICAL METHODS FOR SOLVING THE BOLTZMANN EQUATION}

The Boltzmann equations were solved by direct matrix inversion. The Hall effect and the $B^{2}$-magnetoresistivity were calculated in perturbation theory in $B$. To keep the size of the matrices small it is important to use all the symmetries of the problem and to choose a suitable discretization of the Fermi surface. At low temperatures the distribution function $\Phi_{\mathbf{k}}$ changes very rapidly perpendicular to the hot lines. Therefore we define the distribution function on polygon-shaped patches on the Fermi surface which are relatively long parallel to the hot lines $\Delta k_{\|} \ll k_{F}$ but short in the perpendicular direction close to the hot lines $\Delta k \ll k_{F} \min [\sqrt{t}, t / \sqrt{x}]$. Because the kernel of the integral equation is strongly peaked it is essential to calculate a small number of matrix elements $M_{i j}=\iint_{\text {patch } i} d k \iint_{\text {patch } j} d k^{\prime} G_{k k^{\prime}}$ numerically if they involve a momentum transfers $\mathbf{k}-\mathbf{k}^{\prime}$ close 
to $\mathbf{Q}_{i}$. To speed up the calculations one can approximate $I[y] \approx \pi^{2}(2 \pi+y) /\left(y\left(4 \pi^{2}+6 \pi y+3 y^{2}\right)\right)$ which is asymptotically exact in next-to-leading order for large and small arguments; errors are smaller than $1.5 \%$ (The previously used27,25 approximation $I[y] \approx \pi^{2} /(y(3 y+2 \pi)$ is also sufficient to reproduce next to all of our results with essentially neglegible error, one exception is the temperature dependence of the Hall effect in the ultra-clean limit, where it wrongly predicts $R_{H} \sim c_{1} \pm c_{2} T$ instead of the correct $R_{H} \sim c_{1} \pm c_{2} T^{3 / 2}$ ). We used Fermi surfaces with cubic symmetry. As the largest irreducible representation of the 48-dimensional cubic group is only three dimensional, it was possible to reduce the size of the matrices by the huge factor $16 \times 16$ using the full symmetry of the scattering matrix. We didn't try to solve the Boltzmann equations numerically in a finite magnetic field, which would break the cubic symmetry, but used perturbation theory in $B$ to calculate the figures of this paper.

It is essential to use a proper discretization of the magnetic field term which conserves

$$
\begin{aligned}
& \iint_{S}\left[\mathbf{B} \cdot\left(\mathbf{v}_{\mathbf{k}} \times \nabla_{\mathbf{k}} \Phi_{\mathbf{k}}\right)\right]\left(d^{2} \mathbf{k} / v_{\mathbf{k}}\right) \\
& =\iint_{S}\left(\mathbf{d}^{2} \mathbf{k} \times \nabla_{\mathbf{k}} \Phi_{\mathbf{k}}\right) \cdot \mathbf{B}=\oint_{\partial S} \Phi_{\mathbf{k}}(\mathbf{B} \cdot d \mathbf{k})
\end{aligned}
$$

for an arbitrary part $S$ of the Fermi surface. Otherwise the discretization leads to large errors. To determine the averaged $\mathbf{v} \times \mathbf{B}$ contribution for a single patch, (C1) is applied for a single patch. $\Phi_{\mathbf{k}}$ on the edges of the patch $i$ is determined by a linear interpolation: $\left[\mathbf{B} \cdot\left(\mathbf{v}_{\mathbf{k}} \times\right.\right.$ $\left.\left.\nabla_{\mathbf{k}} \Phi_{\mathbf{k}}\right)\right]\left(d^{2} \mathbf{k} / v_{\mathbf{k}}\right) \approx \sum_{j}\left(\mathbf{B} \cdot \Delta \mathbf{k}_{j}^{i}\right)\left(\bar{\Phi}_{\mathbf{k}_{i, j, 2}}+\bar{\Phi}_{\mathbf{k}_{i, j, 2}}\right) / 2$ where $\Delta \mathbf{k}_{j}^{i}=\mathbf{k}_{i, j, 2}-\mathbf{k}_{i, j, 1}$ describes the $j$ th edge with corners $\mathbf{k}_{i, j, 2}$ and $\mathbf{k}_{i, j, 1}$. The vectors $\Delta \mathbf{k}_{j}^{i}$ point counterclockwise around patch $i$ with $\sum_{j} \Delta \mathbf{k}_{j}^{i}=0$. The value of $\bar{\Phi}_{\mathbf{k}}$ at the corners $\mathbf{k}_{i, j, n}(n=1,2)$ is determined by a simple average over the value of $\Phi$ on each neighboring patch. The details of these averaging procedures are not so important as long as (C1) is not violated.

\section{APPENDIX D: MODELS USED FOR THE NUMERICAL CALCULATION}

For our numerical calculations we use three different models. Model A is based on a spherical Fermisurface (see Fig. 1) and the ordering wave-vector is $\mathbf{Q}=\left(0,0, \pm 2 k_{F} \cos \Theta_{H}\right.$ with $\Theta_{H}=\pi / 6$. The precise value of $\theta_{H}$ are not very important as long as one stays away from $\theta_{H}=0$ ( " $2 k_{F}$ " ordering) or $\theta_{H}=\pi / 2$ (ferramagnetic ordering) where our approach breaks down 16; in our numerical calculations we use $\theta_{H}=\pi / 6$. The dispersion $\omega_{\mathbf{q}}$ in (2) is given by $(\mathbf{q}-\mathbf{Q})^{2} / q_{0}^{2}$.

Model B describes a cubic system with commensurate spin waves with ordering vectors $\mathbf{Q}=( \pm 1, \pm 1, \pm 1)$ and $\omega_{\mathbf{q}}=3+\sum_{i=x, y, z} \cos \pi q_{i}$. The bandstructure was choosen to be featureless but it includes terms which mix the various directions and it is characterized by 8 non-intersecting "hot lines": $\epsilon_{\mathbf{k}}=t \sum_{x, y, z} \cos \pi k_{i}+$ $t^{\prime} \sum_{x, y, z} \cos 2 \pi k_{i}+t^{\prime \prime}\left(\cos \pi k_{x} \cos \pi k_{y}+\cos \pi k_{x} \cos \pi k_{z}+\right.$ $\left.\cos \pi k_{y} \cos \pi k_{z}\right)-\mu$ with $t=-0.2, t^{\prime}=0.05, t^{\prime \prime}=-0.045$ and $\mu=-0.0225$

We have compared model B to model C, where the ordering vector is parallel to the principal axes: $\mathbf{Q}_{i}=$ $( \pm 1,0,0),(0, \pm 1,0),(0,0, \pm 1)$ using also a different bandstructure. All results are very similar compared to those of model B and are therefore not shown in this paper.

${ }^{1}$ P.W. Anderson, The Theory of Superconductivity in High $T_{c}$ Cuprates (Princeton University Press, Princeton, 1997).

${ }^{2}$ S.V. Kravchenko et al., Phys. Rev. B 50 (1994), 8039; S.V. Kravchenko et al., Phys. Rev. B 51 (1995), 7038.

${ }^{3}$ H.v. Löhneysen et al., Phys. Rev. Lett. 72, 159 (1994); H. v. Löhneysen, J. Phys.: Cond. Matt. 8, 9689

${ }^{4} \mathrm{~A}$ good overview is given in the proceedings of the Conference on Non-Fermi-Liquid Behavior in Metals, J. Phys.: Cond. Mat. 8 (1996).

${ }^{5}$ S. R. Julian, et al., J. Phys. Cond. Mat. 8, 9675 (1996).

${ }^{6}$ N. D. Mathur, et al., Nature 394, 39 (1998).

${ }^{7}$ P. Gegenwart et al., Phys. Rev. Lett. 81, 1501 (1998).

${ }^{8}$ F.M. Grosche et al., preprint, cond-mat/9812133.

${ }^{9}$ R. Hauser el al, JMMM 177-181, 292 (1998).

${ }^{10}$ R. Heuser, E.-W. Scheidt, T. Schreiner, and G.R. Stewart , Phys. Rev. B 58, R15959 (1998).

${ }^{11}$ H. Wilhelm et al, preprint, to appear in Rev. H. P. Sci. Techn.

12 O. Trovalli, C. Geibel, F. Steglich, Preprint, submitted to Physica B.

${ }^{13}$ E.J. Feeman et al, Phys. Rev. B 58, 16027 (1998).

${ }^{14}$ S. Doniach, Physica B 91, 231 (1977).

15 J. A. Hertz, Phys. Rev. B 14, 1165 (1976).

16 A. J. Millis, Phys. Rev. B 48, 7183 (1993).

17 T. Moriya and T. Takimoto, J. Phys. Soc. Jpn. 64, 960 (1995); S. Kambe et al., J. Phys. Soc. Japam 65, 3294 (1996).

18 A. Schröder et al., Phys. Rev. Lett. 80, 5623 (1998);

${ }^{19}$ Q. Si, J. L. Smith, and K. Ingersent, cond-mat/9905006. Q. Si and J. L. Smith, cond-mat 9903083.

${ }^{20} \mathrm{P}$. Coleman, preprint, cond-mat/9809436.

${ }^{21}$ M. A. Continentino, J. Phys. I 1, 693 (1991).

22 R.B. Griffiths, Phys. Rev. Lett. 29 (1969), 17; B.M. McCoy and T.T. Wu, Phys. Rev. 176 (1968) 631; ibid. 188, 982 (1969).

23 A. H. Castro Neto, G. Castilla, and B.A. Jones, Phys. Rev. Lett. 813531 (1998) ; M. C. de Andrade et al, Phys. Rev. Lett. 815620 (1998); C-Y Liu et al., preprint, condmat/9909215.

${ }^{24}$ R. Narayanan, T. Vojta, D. Belitz, T. R. Kirkpatrick, condmat/9905047. T. R. Kirkpatrick and D. Belitz, Phys. Rev. Lett. 76, 2571, (1996); ibid. 78, 1197 (1997).

${ }^{25}$ A. Rosch, Phys. Rev. Lett 82, 4280 (1999). 
${ }^{26}$ A. Rosch, preprint, cond-mat/9908245, submitted to Physica B.

${ }^{27}$ R. Hlubina and T. M. Rice, Phys. Rev. B 51, 9253 (1995).

${ }^{28}$ B.L. Atshuler, L.B. Ioffe, A.J. Millis, Phys. Rev. B 52, 5563 (1995).

${ }^{29}$ We do not consider situations as in $\mathrm{CeCu}_{6-x} \mathrm{Au}_{x} 38$ where the spin fluctuations are extremely anisotropic.

${ }^{30}$ In three dimensions the scattering rate of the quasiparticles at the "hot lines" vanishes linear in $T$, therefore the quasiparticle approximation is only "marginally" valid. The situation is less clear in $d=2$, where the scattering rate at "hot spots" 27 is proportional to $\sqrt{T}$.

${ }^{31}$ This definition differs from the one given in 25 by a factor $\pi$ correcting a misprint.

${ }^{32}$ For simple enough band structures in crystals with mirror planes perpendicular to the ordering wave vectors $\mathbf{Q}_{i}$, the points $\mathbf{k}_{H}$ on the hot lines can be mapped on $\mathbf{k}_{H} \pm \mathbf{Q}_{i}$ by a mirror transformation, $v_{\mathbf{k}_{H}}=v_{\mathbf{k}_{H} \pm \mathbf{Q}_{i}}$ and $\alpha_{H}$ is 1

${ }^{33} \mathbf{k} \pm \mathbf{Q}_{i}$ is projected on the Fermi surface, $\pm \mathbf{Q}_{i}$ is choosen in such a way that $\mathbf{k} \pm \mathbf{Q}_{i}$ is close to the Fermi surface. These details are not important, because $p_{\mathbf{k}}$ vanishes far from the hot lines. We also neglect points on the Fermi surface where two hot lines cross, their contribution is small.

${ }^{34}$ A. Chubukov and D. Morr, Phys. Rev. Lett. 81, 4716 (1998).

35 B. P. Stojkovic and D. Pines, Phys. Rev. Lett. 76, 811 (1996); B. P. Stojkovic and D. Pines, Phys. Rev. B 55, 8576 (1997).

${ }^{36}$ B.L. Altshuler and A.G. Arononv, in Electron-Electron Interaction in Disordered Systems, edited by A.L. Efros and M. Pollak (North Holland, Amsterdam, 1985).

${ }^{37}$ H. Kontani, K. Kanki, and K. Ueda, preprint, condmat/9905427; K. Kanki, H. Kontani, preprint, condmat/9905428

${ }^{38}$ A. Rosch et al., Phys. Rev. Lett. 79, 159 (1997); O. Stockert et al., Phys. Rev. Lett. 80, 5627 (1998);

${ }^{39}$ L.B. Ioffe and A.J. Millis, Phys. Rev. B 51, 16151 (1995).

${ }^{40}$ P. Coleman, C. Pépin, and A.M. Tsvelik, preprint, condmat/9909073.

${ }^{41}$ In many heavy-Fermion systems, skew scattering dominates the Hall effect. This is not considered in this paper. See for example H. Kontani and K. Yamada, J. Phys. Soc. J. 63, 2627 (1994). P. Coleman, P.W. Anderson and T.V. Ramakrishman, Phys. Rev. Lett. 55, 414 (1985).

42 J. Ziman, Electrons and Phonons, Clarendon, Oxford (1960). 